\title{
HECTD3 mediates TRAF3 polyubiquitination and type I interferon induction during bacterial infection
}

\author{
Fubing Li, ${ }^{1}$ Yang Li, ${ }^{1,2}$ Huichun Liang, ${ }^{1}$ Tao Xu, ${ }^{1,2}$ Yanjie Kong, ${ }^{1}$ Maobo Huang, ${ }^{1}$ Ji Xiao, ${ }^{3}$ Xi Chen, ${ }^{1}$ Houjun Xia, ${ }^{1}$ Yingying Wu, ${ }^{1}$ \\ Zhongmei Zhou, ${ }^{1}$ Xiaomin Guo, ${ }^{1,2}$ Chunmiao Hu, ${ }^{1,2}$ Chuanyu Yang, ${ }^{1}$ Xu Cheng, ${ }^{4}$ Ceshi Chen, ${ }^{1}$ and Xiaopeng Qi',2 \\ 'Key Laboratory of Animal Models and Human Disease Mechanisms of the Chinese Academy of Sciences and Yunnan Province, and 'Key Laboratory of Bioactive Peptides of Yunnan Province, Kunming \\ Institute of Zoology, Chinese Academy of Sciences, Kunming, Yunnan, China. ${ }^{3}$ State Key Laboratory of Respiratory Disease, Guangzhou Institute of Respiratory Disease, The First Affiliated Hospital of \\ Guangzhou Medical University, Guangzhou, China. ${ }^{4}$ Department of Pharmacology, College of Pharmaceutical Sciences, Soochow University, Suzhou, China.
}

\begin{abstract}
Lysine-63-linked (K63-linked) polyubiquitination of TRAF3 coordinates the engagement of pattern-recognition receptors with recruited adaptor proteins and downstream activator TBK1 in pathways that induce type I IFN. Whether autoubiquitination or other E3 ligases mediate K63-linked TRAF3 polyubiquitination remains unclear. We demonstrated that mice deficient in the E3 ligase gene Hectd3 remarkably increased host defense against infection by intracellular bacteria Francisella novicida, Mycobacterium, and Listeria by limiting bacterial dissemination. In the absence of HECTD3, type I IFN response was impaired during bacterial infection both in vivo and in vitro. HECTD3 regulated type I IFN production by mediating K63-linked polyubiquitination of TRAF3 at residue K138. The catalytic domain of HECTD3 regulated TRAF3 K63 polyubiquitination, which enabled TRAF3-TBK1 complex formation. Our study offers insights into mechanisms of TRAF3 modulation and provides potential therapeutic targets against infections by intracellular bacteria and inflammatory diseases.
\end{abstract}

\section{Introduction}

The high prevalence of multidrug-resistant bacterial infections is a growing public health threat and can complicate further treatment, especially for infections by intracellular bacterial pathogens, which can establish an intracellular replicative niche and promote antibiotic resistance $(1,2)$. Hence, new therapeutic approaches are required to mount effective immune responses against bacterial pathogens. The type I IFN (IFN-I) signaling pathway serves pleiotropic functions in inducing the programs essential for host defense against pathogenic infections while limiting tissue damage and preventing autoimmunity. Thus, type I IFN production is tightly controlled to ensure appropriate immune responses $(3,4)$. Type I IFN production is initiated through detection of conserved molecular patterns by germline-encoded host pattern-recognition receptors (PRRs) (5). Stimulation of membrane-bound TLR4 by LPS and of endosomal TLR3 by dsRNAs results in TIR domaincontaining adapter-inducing IFN- $\beta$-dependent (TRIF-dependent) type I IFN induction (6). Recognition of cytosolic RNA by retinoic acid inducible gene-I (RIG-I) and its homolog melanoma differentiation-associated gene 5 (MDA5) induces mitochondrial antiviral signaling-mediated (MAVS-mediated) TBK1 activation and IRF3 phosphorylation, which lead to type I IFN production (7). In contrast, engagements of cytosolic sensors cyclic GMP-AMP synthase (cGAS), DNA-dependent activator of IRFs (DAI), IFN- $\gamma-$ inducible protein 16 (IFI16), or DEAD box polypeptide 41 (DDX41)

Authorship note: FL, YL, HL, and TX contributed equally to this work. CC and XQ are co-senior authors.

Conflict of interest: The authors have declared that no conflict of interest exists. Submitted: February 8, 2018; Accepted: June 14, 2018.

Reference information: J Clin Invest. 2018;128(9):4148-4162.

https://doi.org/10.1172/JCI120406. with dsDNA trigger the activation of TBK1 and phosphorylation of IRF3 in a stimulator of IFN genes-dependent (STING-dependent) manner $(8,9)$. Therefore, signaling pathways for TLR3, TLR4, RIG-I, MDA5, and cytosolic DNA sensors converge at the level of TBK1 activation to induce type I IFN production (10).

Ubiquitin modifications of critical adaptors and regulators in PRR signaling cascades play pivotal roles in modulating type I IFN induction and coordinating an efficient immune response. E3 ubiquitin ligases harbor distinct protein interaction motifs that help determine substrate specificity and are mainly categorized as homologous to E6-AP carboxyl terminus (HECT) and really interesting new gene (RING) domain-containing E3 ligases. RING-type E3 ligases meditate the transfer of ubiquitin directly from E2 ubiquitin to the substrate (11), whereas HECT family members possess intrinsic catalytic activity and presumably serve as catalytic intermediates in ubiquitination via a ubiquitinHECT thioester complex (12-14).

Upon infection with DNA or RNA viruses, several members of RING-like E3s can play positive or negative roles in mediating type I IFN production through various types of ubiquitination of cytosolic sensors and adaptors (15-17). For example, MIB1, MIB2, NRDP1, and RNF128 mediate Lys63-linked ubiquitination of TBK1 $(10,18,19)$; TRIM31 regulates MAVS aggregation via Lys63-linked polyubiquitination (20); TRIM25 and MEX3C induce Lys63-linked ubiquitination of RIG-I (21, 22); TRIM65 mediates Lys63-linked ubiquitination of MDA5 (23); TRIM56 and TRIM32 target STING for Lys63-linked ubiquitination $(24,25)$; AMFR catalyzes Lys27-linked polyubiquitination of STING (26); and RNF26 promotes Lys11-linked polyubiquitination of STING (27). All these ubiquitination mechanisms stabilize the targets and trigger type I IFN production, which in turn elicits host antiviral innate immunity. On the other hand, RNF125 regulates 
ubiquitination of RIG-I and MDA5 (28); RNF5 and TRIM30 $\alpha$ mediate Lys48-linked ubiquitination of STING $(29,30)$; NLRP4mediated DTX4 and TRIP induce Lys48-linked polyubiquitination of TBK1 $(31,32)$; and TRIM26 promotes Lys48-linked polyubiquitination of IRF3 (33), which all cause proteasomal degradation of targets and negative regulation of type I IFN production and antiviral signaling.

Despite advances in studying the role of E3 ubiquitin ligases in type I IFN production during viral infection, the molecular mechanisms underlying type I IFN induction during bacterial infection and the role of the HECT family of E3 ligases in the innate immune response remain unknown. Previous work by us and others demonstrated that a recently defined HECT family member, HECTD3, was highly expressed in certain tumor cells and potentially facilitated cancer cell survival and proliferation by mediating the stability of several target proteins (e.g., MALT1, caspase- 8 , caspase- 9 , syntaxin 8 , and Tara), but the physiological relevance of most of these interactions remains to be defined $(13,34,35)$. However, the role of HECTD3 in innate immunity is unknown. Herein, we report a new pathway of innate immunity in which HECTD3 acts as a crucial regulator for the expression of cytokine genes by controlling the activity of TRAF3. HECTD3 was crucial to regulating TRAF3 lysine-63-linked (K63-linked) polyubiquitination and type I IFN production.

\section{Results}

HECTD3 plays negative roles in host defense against $F$. novicida infection. The lack of classical virulence factors in facultative intracellular bacteria, such as Francisella, Mycobacterium, and Listeria, helps them evade the hostile host environment and establish persistent

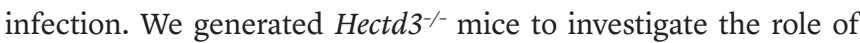
HECTD3 in host defense against infection by intracellular bacteria (Supplemental Figure 1, A-C; supplemental material available online with this article; https://doi.org/10.1172/JCI120406DS1). $\mathrm{Hectd}^{-/-}$mice were viable and had immune cell compositions, including neutrophils, macrophages, dendritic cells, $\mathrm{CD}^{+} \mathrm{T}$ cells, $\mathrm{CD}^{+} \mathrm{T}$ cells, B cells, and basophils in $\mathrm{BM}$, spleen, and peripheral blood, that were similar to those of WT mice (Supplemental Figure $1, \mathrm{D}-\mathrm{G})$, indicating that HECTD3 did not regulate immune celllineage commitment and hematopoiesis. WT and Hectd $3^{-/-}$mice were subcutaneously infected with a lethal dose $\left(3.0 \times 10^{5} \mathrm{CFUs}\right)$ of $F$. novicida, and survival was monitored over time. WT mice exhibited more severe symptoms of illness, such as ruffled fur and hunched back, at day 2 after infection (Supplemental Figure 2A) and entirely succumbed by day 6 , whereas $75 \%$ of $\mathrm{Hectd}^{-/-}$mice survived beyond day 10 (Figure 1A). WT mice also lost more body weight than did $\mathrm{Hectd}^{-/-}$mice by day 4 after infection (20\% vs. $5 \%$ of starting body weight, respectively) (Figure 1B). To determine whether reduced mortality in Hectd $3^{-/-}$mice was due to less bacterial burden, we enumerated the number of bacteria in the spleen, liver, and lung of WT and Hectd3 $3^{-/-}$mice after 2 days of infection with F. novicida. Bacterial loads in the spleen, liver, and lung of $\mathrm{Hectd3}^{-/}$mice were significantly lower than those in WT mice (Figure 1C). H\&E staining and anti-Ly-6G immunohistochemical staining showed less inflammation and lower neutrophil infiltration, respectively, in the spleen and liver of infected Hectd $3^{-/-}$mice than of WT mice (Figure 1, D and E). In line with these findings, reduced bacterial burden in $H e c t d 3^{-/-}$mice was also associated with lower expression of genes encoding inflammatory cytokines Tnfa, Il6, and Ifn $b$ and lesser production of TNF- $\alpha$, IL- 6 , and IFN- $\beta$ than in WT mice (Figure 1, F and G). Further, production of inflammatory cytokines TNF- $\alpha$, IL- 6 , IL- $1 \beta$, and IFN- $\beta$ in the peripheral blood was significantly lower in Hectd $3^{-/-}$mice than in WT mice (Supplemental Figure 2B). Strikingly, activation of NF- $\kappa$ B indicated by IкB $\alpha$ phosphorylation and type I IFN downstream molecules STAT1 and STAT3 was substantially reduced in $\mathrm{Hectd}^{-/-}$mice at day 2 after infection (Figure $1 \mathrm{H}$ ). In addition, multiple cell-death pathways associated with the expression of ZBP1 and caspase-11 and activation of caspase- 3 and -1 were decreased in the liver of $\mathrm{Hectd}^{-/}$mice at day 2 after infection (Figure $1 \mathrm{H}$ ). Collectively, these results indicate that HECTD3 negatively regulates host defense against $F$. novicida infection.

Hectd3-deficient mice are resistant to Mycobacterium and Listeria infection. To examine whether the protective role of HECTD3 deficiency in host defense was specific to $F$. novicida infection, we infected WT and Hectd3 $3^{--}$mice with GFP-expressing Mycobacterium bovis bacillus Calmette-Guérin (BCG-GFP) and Listeria monocytogenes separately. After intranasal administration of BCG-GFP, the bacterial burden, indicated by the presence of BCG genomic DNA in the lung, was significantly lower in $\mathrm{Hectd3}^{-/-}$mice than in WT mice (Figure 2A). Reduced BCG-GFP load in the lung of Hectd3 $3^{-/-}$ mice was also confirmed by CFU analysis (Figure 2B). In line with this, $H \& E$ staining revealed that infiltration of immune cells into the lung of WT mice was much higher than into that of Hectd3 $3^{--}$mice (Figure 2C). After intraperitoneal infection with L. monocytogenes, $\mathrm{Hectd}^{-/-}$mice lost less body weight and exhibited lower bacterial burden in the spleen and liver than did WT mice (Figure 2, D and E). Reduced bacterial burden in $\mathrm{Hectd3}^{-/-}$mice was associated with decreased infiltration of immune cells into the liver and expression of inflammatory cytokine genes Tnfa, Il6, and Ifnb (Figure 2, F and $G)$. Moreover, cell death induction, indicated by increased levels of caspase-3 and -11 and ZBP1 activation after L. monocytogenes infection in the liver, was substantially lower for Hectd3 $3^{-/}$mice than for WT mice (Figure 2H). To determine the role of HECTD3 in host defense against infection by extracellular bacteria, we intraperitoneally infected WT and Hectd $3^{--}$mice with E. coli. In contrast to what was observed in response to L. monocytogenes infection, Hectd3 $3^{-/-}$ and WT mice displayed similar losses in body weight and bacterial burden in the spleen and liver (Figure 2, I and J). Production of inflammatory cytokines IL- 6 and TNF- $\alpha$ was also comparable between WT and $\mathrm{Hectd}^{-/-}$mice (Figure 2K), indicating that HECTD3 does not play negative roles in host defense against $E$. coli infection. Taken together, these results suggest that Hectd3 $3^{--}$mice are specifically resistant to infection by intracellular bacteria.

HECTD3 deficiency limits F. novicida dissemination through bacteria-carrying cells. Macrophages and neutrophils are important components of the innate immune system and primary immune cells recruited to sites of infection, which control microbe dissemination during the early phase of infection (36). To investigate the protective role of $\mathrm{Hectd}^{-/-}$mice in response to $F$. novicida infection, we analyzed bacterial dissemination through intranasal infection with GFP-expressing F. novicida, in which initial bacterial load can be measured. In line with results from subcutaneous infection with $F$. novicida (Figure 1), 
A

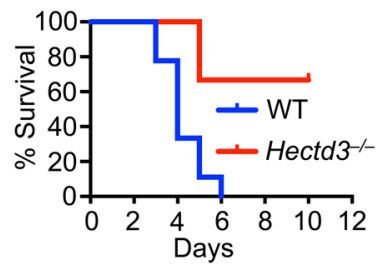

C
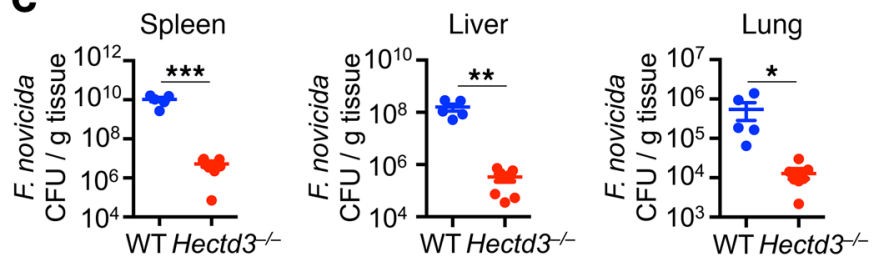

D

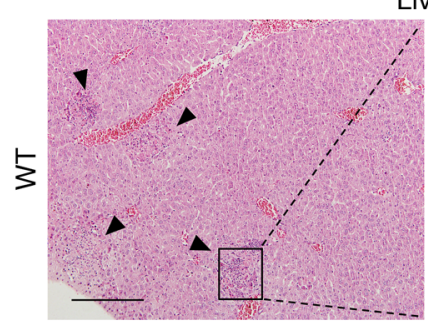

Liver
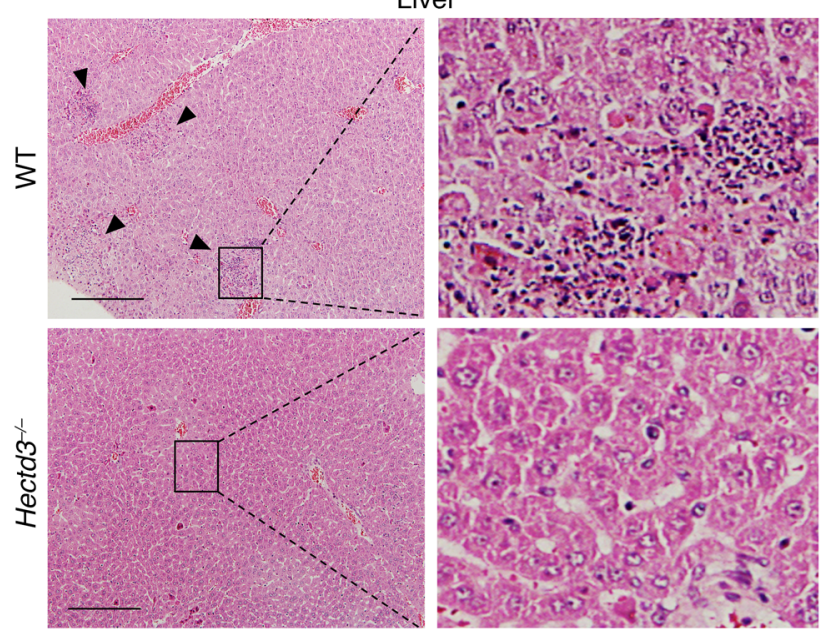

E
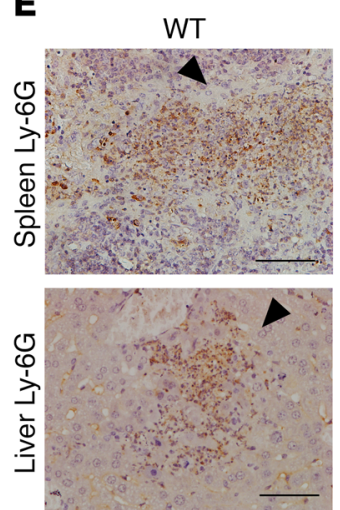

B

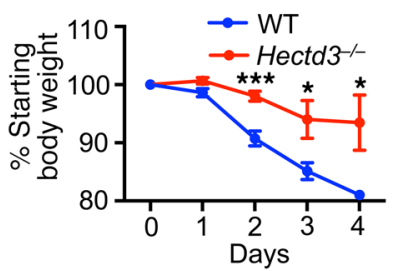

H

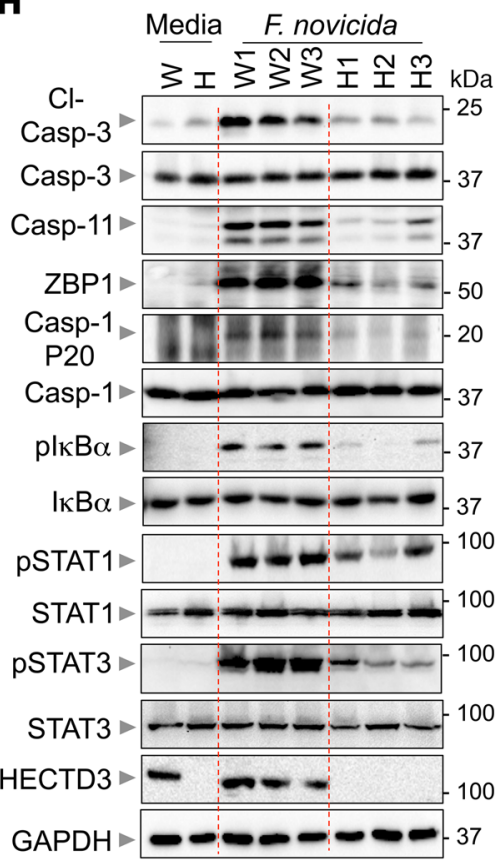

$\mathbf{F}$
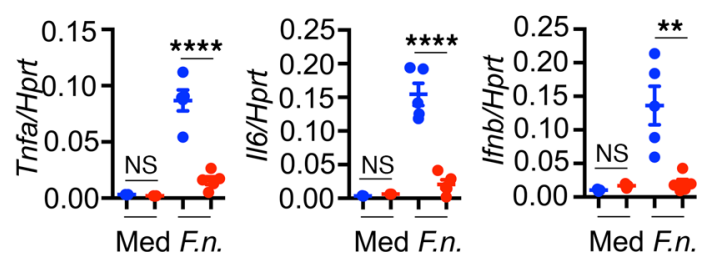

G

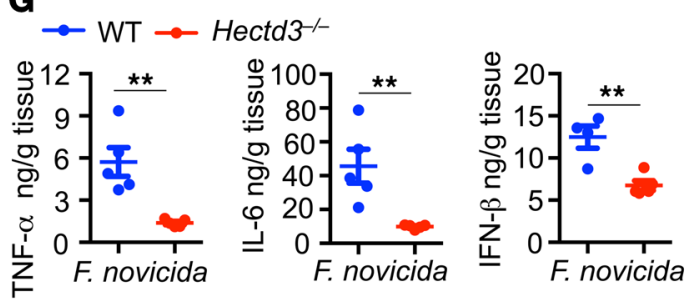

Figure 1. Hectd3 $3^{-/-}$mice are resistant to F. novicida infection. (A) Hectd3 $3^{-/-}$mice $(n=9)$ and littermate WT controls $(n=9)$ were infected subcutaneously with $3.0 \times 10^{5} \mathrm{CFUs}$ of $F$. novicida, and survival was monitored. (B) Body weight of WT and Hectd $3^{-/-}$mice after F. novicida infection as in A, presented relative to the starting body weight at day 0 , which was set as $100 \%$. (C) Hectd3 $3^{--}$mice and littermate WT controls were infected subcutaneously with $3.0 \times 10^{5}$ CFUs of $F$. novicida, and bacterial burden in the spleen, liver, and lung on day 2 after infection was measured. (D) H\&E staining of liver sections from WT and Hectd3 $3^{-/}$mice on day 2 after infection with F. novicida. Arrowheads indicate infiltrated immune cells. (E) Ly-6G immunohistochemical staining of spleen and liver sections from WT and Hectd3 $3^{-/-}$mice on day 2 after infection with F. novicida. Arrowheads indicate neutrophil recruitment. Scale bars: $50 \mu \mathrm{m}$. (F and $\mathbf{G})$ Expression of genes encoding TNF- $\alpha$, IL-6, and IFN- $\beta$ (F) and production of TNF- $\alpha$, IL-6, and IFN- $\beta$ (G) were analyzed in liver tissues from WT and Hectd $3^{-1-}$ mice on day 2 after infection with F. novicida (F.n.). (H) Liver tissue samples from WT (W1, W2, and

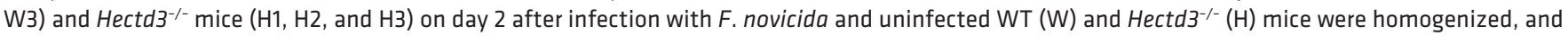
lysates were analyzed for activation and expression of caspase-3, caspase-1, caspase-11, ZBP1, and HECTD3, and phosphorylation of IкB $\alpha$, STAT1, and STAT3. GAPDH was used as loading control. Each symbol indicates an individual mouse (C, F, and $\mathbf{G})$. Data represent 3 independent experiments and are presented as mean \pm SEM. ${ }^{*} P<0.05 ;{ }^{* *} P<0.01 ;{ }^{* *} P<0.001 ;{ }^{* * * *} P<0.0001$. 
A

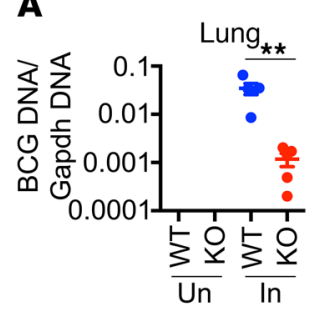

D

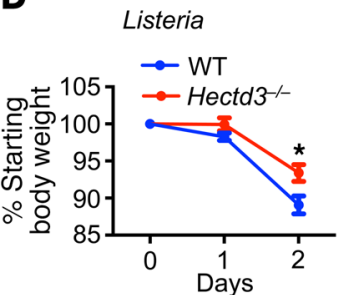

E
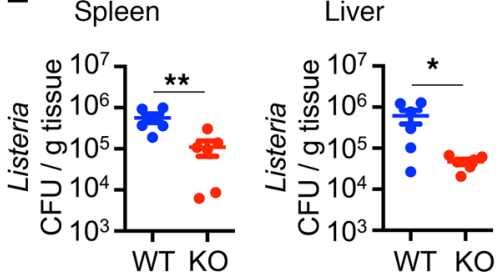

C

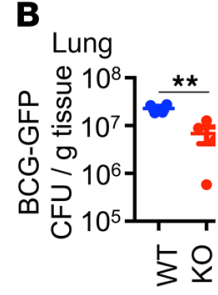

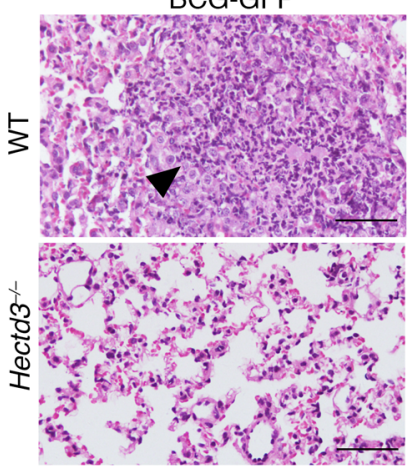

F Listeria

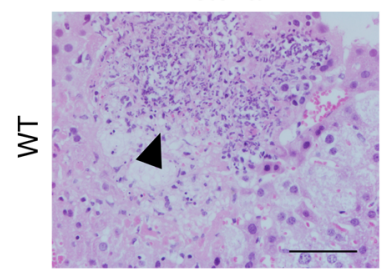

\section{G}
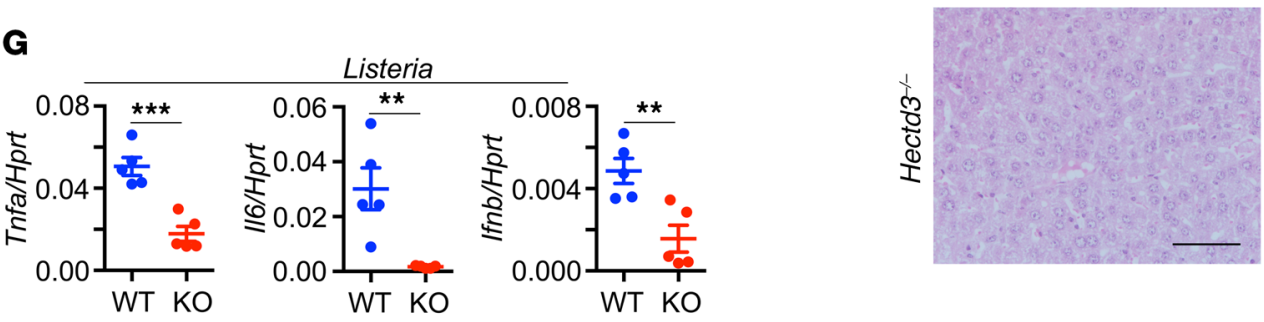

H

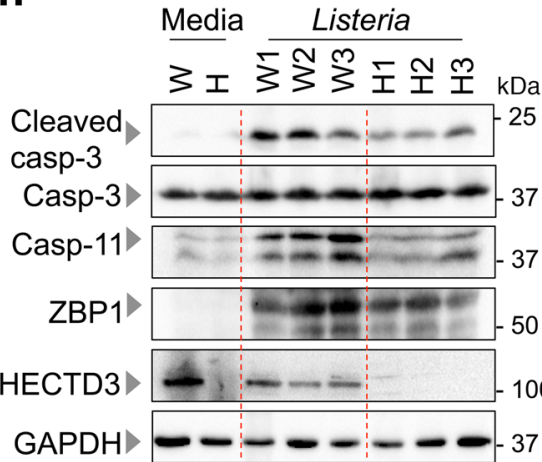

I

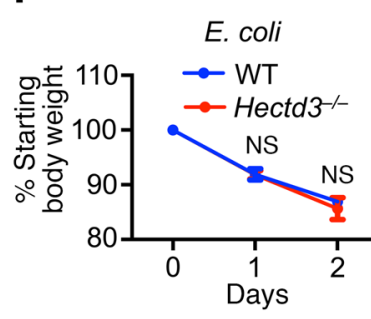

J

Spleen Liver
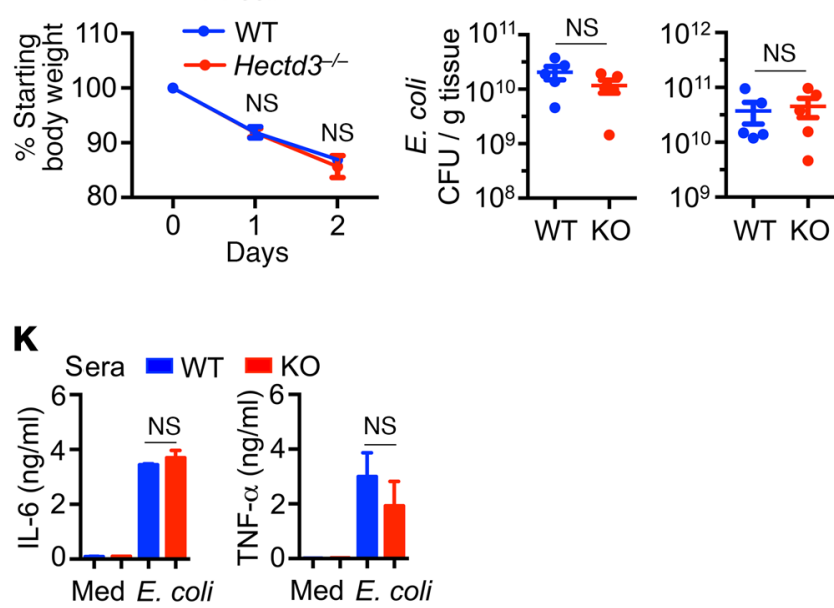

Figure 2. HECTD3 deficiency is protective against Mycobacterium and Listeria infections, but not $\mathbf{E}$. coli infection. (A-C) Hectd3 $3^{-/-}$(KO) mice and littermate WT controls were intranasally infected with GFP-expressing BCG (7.5 $\times 10^{6}$ CFUs per mouse), and bacterial burden in the lung on day 2 was determined by BCG genomic DNA PCR (A) and CFU analysis (B). H\&E staining of lung sections from WT and Hectd3 $3^{-1-}$ mice on day 2 after infection (C). Arrowhead indicates infiltrated immune cells. (D-H) Hectd3 $3^{-/}$mice and littermate WT controls were intraperitoneally infected with L. monocytogenes (6.0 $\times$ $10^{4}$ CFUs per mouse). Loss in body weight was determined (D), and bacterial burden in the spleen and liver was analyzed on day 2 after infection (E). (F) H\&E staining of liver sections from WT and Hectd $3^{-/-}$mice on day 2 after infection with L. monocytogenes. Arrowhead indicates infiltrated immune cells. (G) Expression of genes encoding TNF- $\alpha$, IL-6, and IFN- $\beta$ was analyzed in liver tissues from WT and Hectd $3^{-/-}$mice on day 2 after infection with $L$. monocytogenes. (H) Liver tissue samples from WT and Hectd $3^{-1-}$ mice on day 2 after infection with $L$. monocytogenes were homogenized, and lysates were analyzed for activation and expression of caspase-3, caspase-11, ZBP1, and HECTD3. CAPDH was used as loading control. (I-K) Hectd3 ${ }^{-1-}$ mice and littermate WT controls were intraperitoneally infected with $E$. coli $\left(1.0 \times 10^{8} \mathrm{CFU}\right.$ s per mouse), and loss in body weight was determined (I), bacterial burden in the spleen and liver was analyzed on day 2 after infection (J), and production of TNF- $\alpha$ and IL-6 (K) was analyzed from WT and Hectd $3^{-1-}$ mice on day 2 after infection with E. coli. Each symbol indicates an individual mouse (A, B, E, G, and J). Data represent 2 independent experiments and are presented as mean \pm SEM. ${ }^{*} P<0.05 ;{ }^{* *} P<0.01 ;{ }^{* *} P<0.001$. Scale bars: $50 \mu \mathrm{m}$. 
A

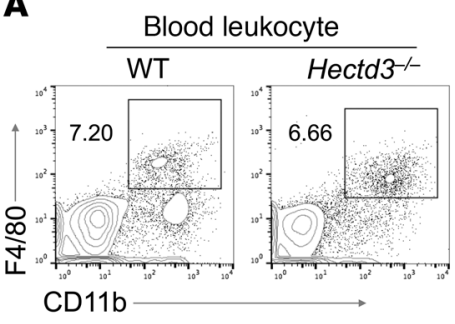

B

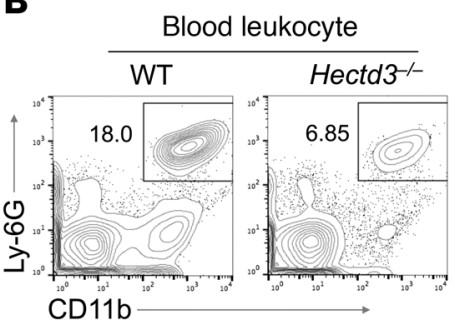

c

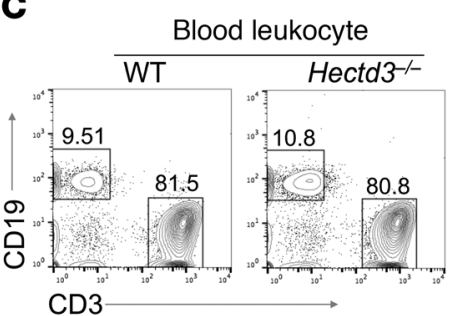

D

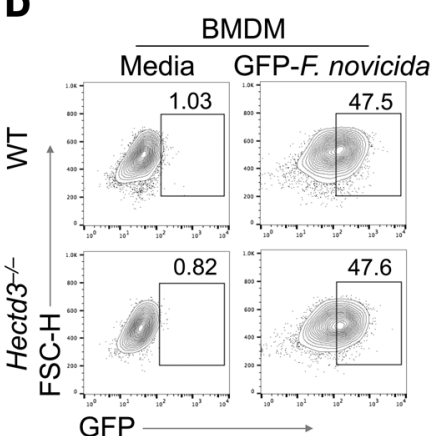

E

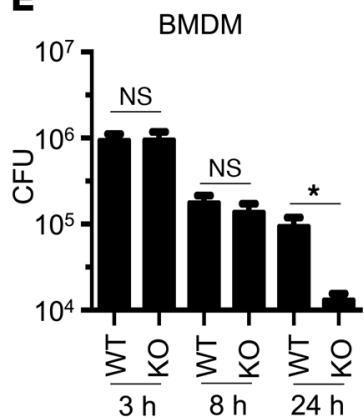

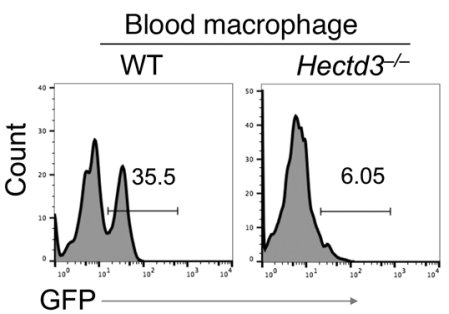

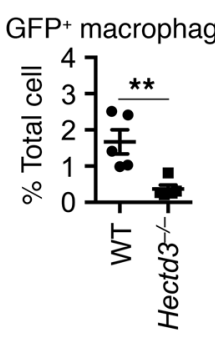

$\mathrm{GFP}^{+}$neutrophil
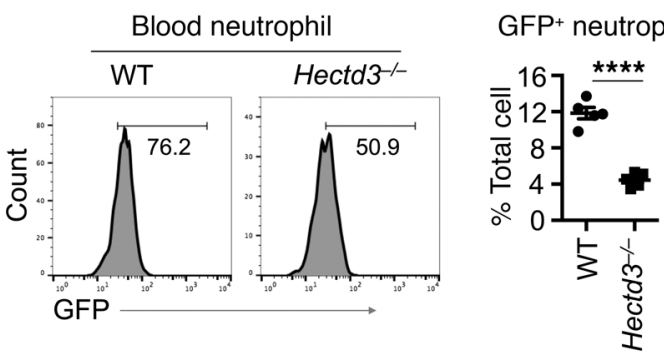
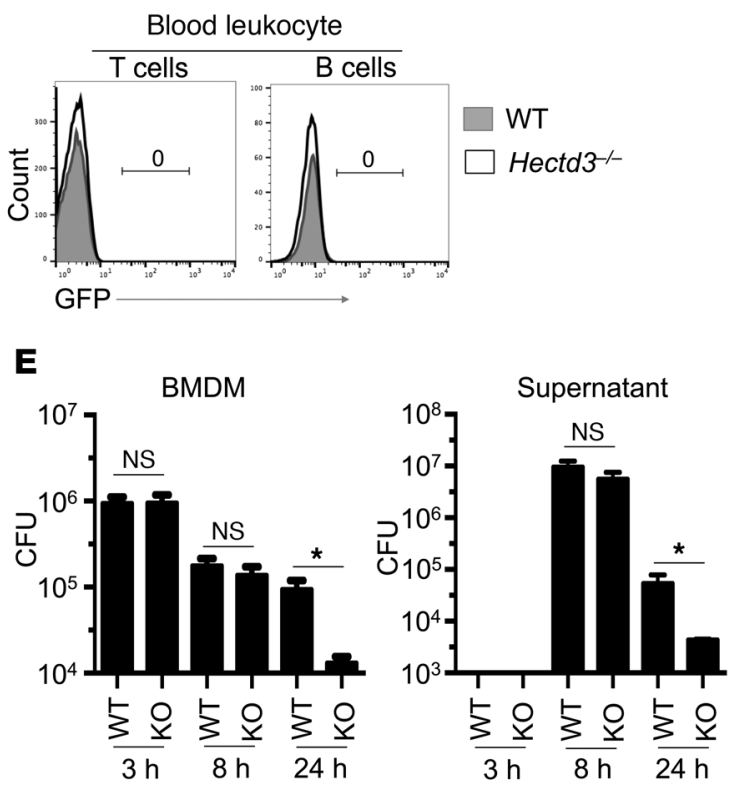

Figure 3. Hectd3 ${ }^{-/-}$mice have fewer $F$. novicida-infected macrophages and neutrophils in the peripheral blood. (A) $\mathrm{Hectd}^{-1-}$ mice and littermate WT controls were intranasally infected with GFPexpressing $F$. novicida $(5,000$ CFUs per mouse) for 12 hours. GFP+ macrophages in blood were analyzed by flow cytometry (left), and the percentage of GFP+ $\mathrm{mac}-$ rophages was quantified (right). (B) GFP+ neutrophils in the blood were analyzed by flow cytometry (left) from mice as in $\mathbf{A}$, and the percentage of GFP ${ }^{+}$neutrophils in the blood was quantified (right). (C) GFPexpressing $F$. novicida-infected $B$ cells and $T$ cells were analyzed in blood leukocytes from mice as in A. (D) BMDMs from WT and $\mathrm{Hectd}^{-/-}$mice were infected with GFP-expressing F. novicida (100 MOI) for 3 hours, and $\mathrm{GFP}^{+} \mathrm{BMDMs}$ were analyzed by flow cytometry. (E) BMDMs from WT and $\mathrm{Hectd}^{-1-}$ mice were infected with $F$. novicida (10 $\mathrm{MOI}$ ) for 3 hours, and numbers of intracellular (BMDM) and extracellular (supernatant) bacteria were enumerated at indicated times. Each symbol indicates an individual mouse (A and $\mathbf{B}$ ). Data represent 3 independent experiments and are presented as mean \pm SEM. ${ }^{*} P<0.05$; ${ }^{*} P<0.01 ;{ }^{* * *} P<0.0001$ bacterial burden of F. novicida at day 3 in the peripheral blood and spleen of Hectd3 $3^{--}$mice was much lower than that of WT mice, but the burden in lungs was comparable, indicating that $\mathrm{Hectd3}^{-/-}$mice were also protected from intranasal infection with F. novicida (Supplemental Figure 3, A-C). Twelve hours after intranasal infection with GFP-expressing $F$. novicida, bacterial burden in the lung was comparable between WT and

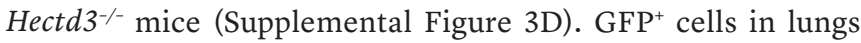
comprised mostly neutrophils and macrophages, but not $\mathrm{B}$ cells and $\mathrm{T}$ cells, indicating that $F$. novicida primarily infected macrophages and neutrophils, which is consistent with results of a previous study (Supplemental Figure 3, E-G, and ref. 37). Notably, infiltration of macrophages and neutrophils into the lung triggered by infection with GFP-expressing F. novicida was comparable between WT and Hectd $3^{--}$mice. Further, in both WT and Hectd $3^{-/}$mice, GFP-expressing F. novicida infected approximately half the macrophages and nearly all neutrophils (Supplemental Figure 3, E and F).
To determine the extent of bacterial dissemination, we further analyzed $\mathrm{GFP}^{+}$macrophages and neutrophils in peripheral blood cells. Interestingly, the percentage of macrophages infected with GFP-expressing F. novicida in circulating blood leukocytes of WT mice was significantly higher than that in those of Hectd $3^{-/-}$mice $(1.67 \% \pm 0.33 \%$ vs. $0.37 \% \pm 0.11 \%$, Figure $3 \mathrm{~A})$. Also, more neutrophils were infected with GFP-expressing F. novicida in WT mice than in $\mathrm{Hectd}^{--}$mice $(11.85 \% \pm 0.63 \%$ vs. $4.46 \% \pm 0.35 \%$, Figure 3B). However, infection by GFP-expressing F. novicida was barely detectable in peripheral blood B cells or $\mathrm{T}$ cells (Figure 3C). Thus, HECTD3 promotes $F$. novicida transmission in vivo by facilitating the migration and dissemination of $F$. novicida-infected macrophages and neutrophils.

To examine whether HECTD3 deficiency affects $F$. novicida infection, we infected WT and Hectd $3^{-/}$BM-derived macrophages (BMDMs) with GFP-expressing F. novicida for 3 hours, and $\mathrm{GFP}^{+}$BMDMs were analyzed by flow cytometry. The percentage of GFP-expressing BMDMs was comparable between WT and 
A
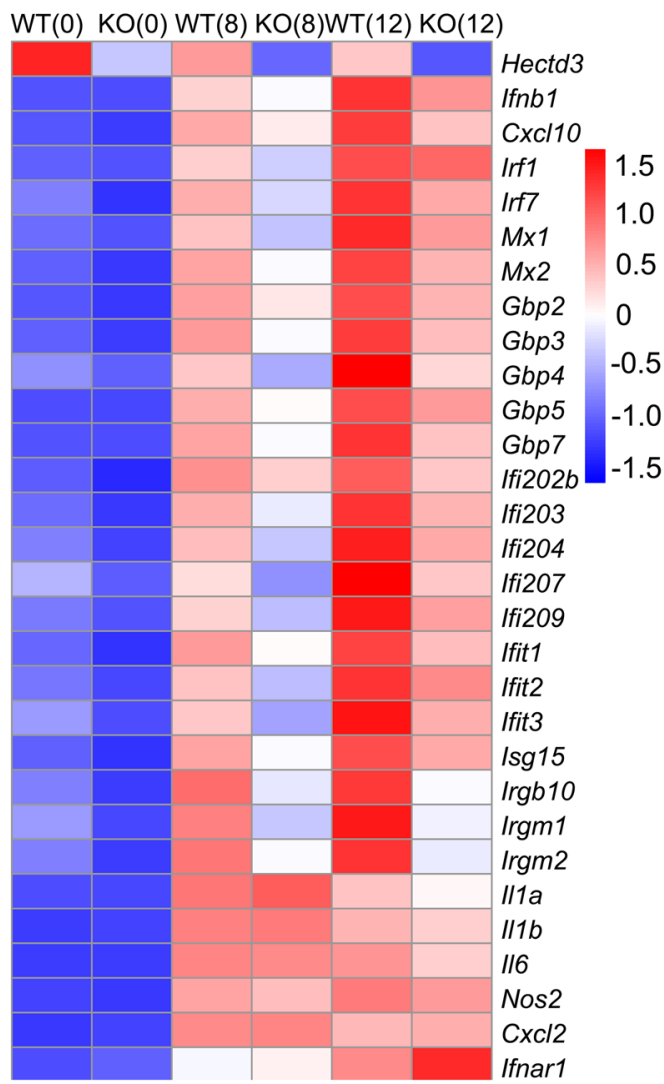

B
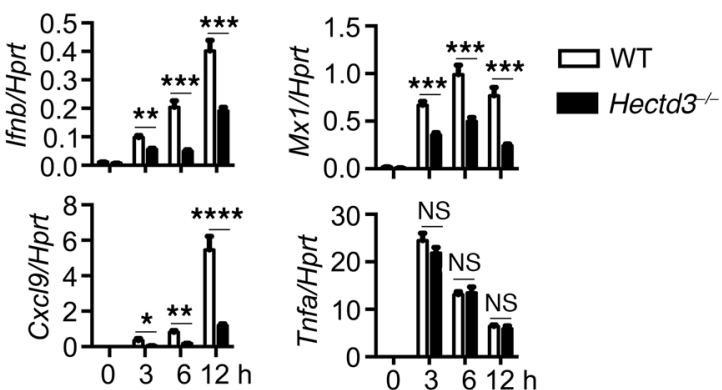

C
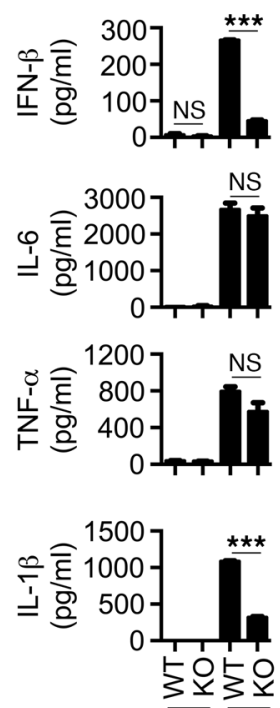

$\overline{\text { Med }} \overline{\text { F.n. }}$
D

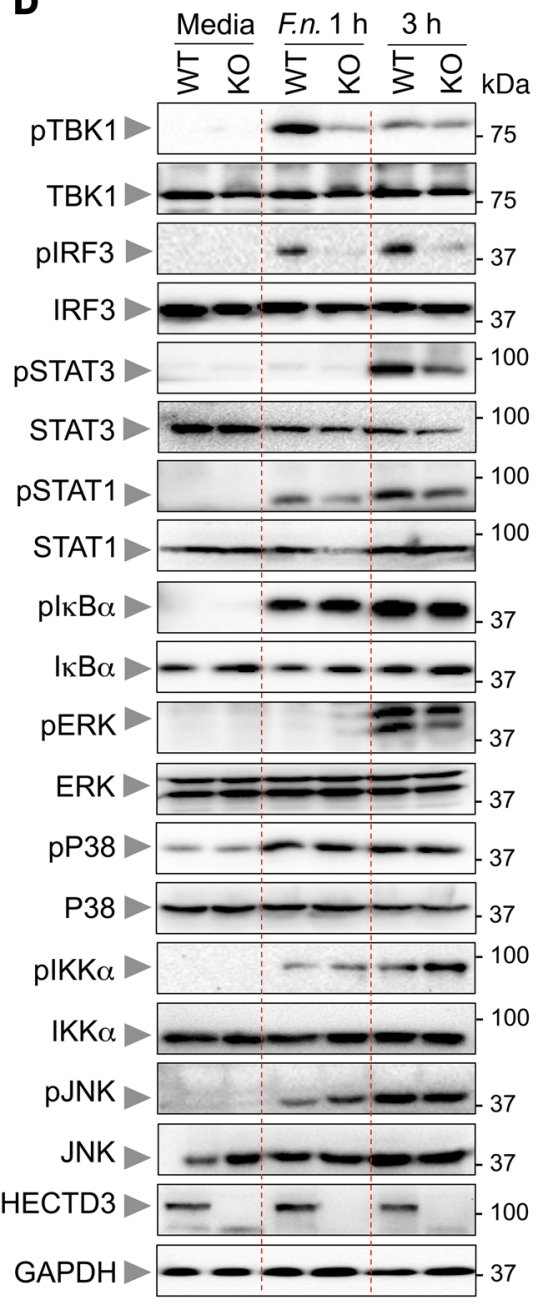

Figure 4. Type I IFN production is impaired in the absence of HECTD3 response to $F$. novicida. (A) RNA-seq analysis of the expression of genes in uninfected and F. novicida-infected WT and Hectd3 $3^{-1-}$ BMDMs for 8 and 12 hours, respectively. Heatmap showing the expression of genes responsive to type I

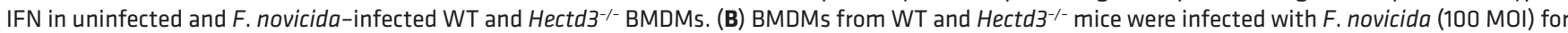
indicated times, and expression of Ifnb, CxcI9, Mx1, and Tnfa was analyzed by qRT-PCR. (C) Production of IFN- $\beta$, IL-6, TNF- $\alpha$, and IL-1 $\beta$ in uninfected (Med) and F. novicida-infected WT and Hectd3 $3^{--}$BMDMs for 14 hours. (D) Immunoblot analysis of phosphorylation of TBK1, IRF3, STAT3, STAT1, IKB $\alpha$, ERK, P38, IKK $\alpha$, and JNK in uninfected and F. novicida-infected WT and Hectd3 $3^{-1-}$ BMDMs at indicated times. Data represent 3 independent experiments (B-D) and are presented as mean \pm SEM. ${ }^{*} P<0.05 ;{ }^{* *} P<0.01 ;{ }^{* *} P<0.001 ;{ }^{* * *} P<0.0001$.

Hectd3 $3^{--}$BMDMs (Figure 3D). To further investigate whether HECTD3 regulates the intracellular replication of F. novicida, WT and Hectd $3^{-/-}$BMDMs were infected with F. novicida and bacterial killing activity was assessed. Strikingly, bacterial growth was significantly inhibited in the absence of HECTD3 24 hours after infection (Figure 3E), indicating that Hectd $3^{-/-}$BMDMs are more efficient than WT BMDMs in suppressing intracellular replication of $F$. novicida.

HECTD 3 regulates type I IFN production. To determine the mechanism by which HECTD3 regulates host defense against infection by intracellular bacteria, we performed genome-wide expression analysis to profile differentially expressed genes in WT and Hectd $3^{--}$BMDMs infected with F. novicida. Hectd $3^{--}$and WT BMDMs had similar expression profiles under normal conditions, indicating that HECTD3 deficiency does not affect BMDM differentiation and maturation (Supplemental Figure 4A and Supplemental Table 1). Of 833 and 790 genes significantly upregulated ( $\geq 2$ fold) in WT and Hectd3 ${ }^{--}$BMDMs, respectively, after F. novicida infection for 8 hours (Supplemental Figure 4, B and C, and Supplemental Table 1), 46 genes were induced in WT BMDMs, but 
A
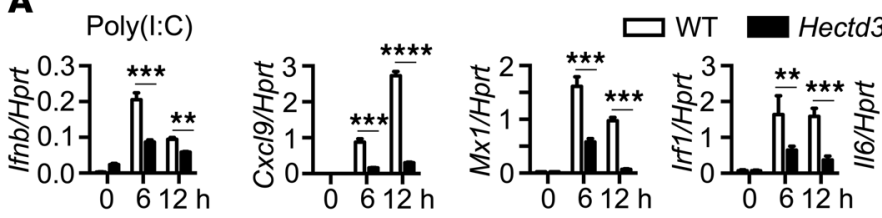

0.03
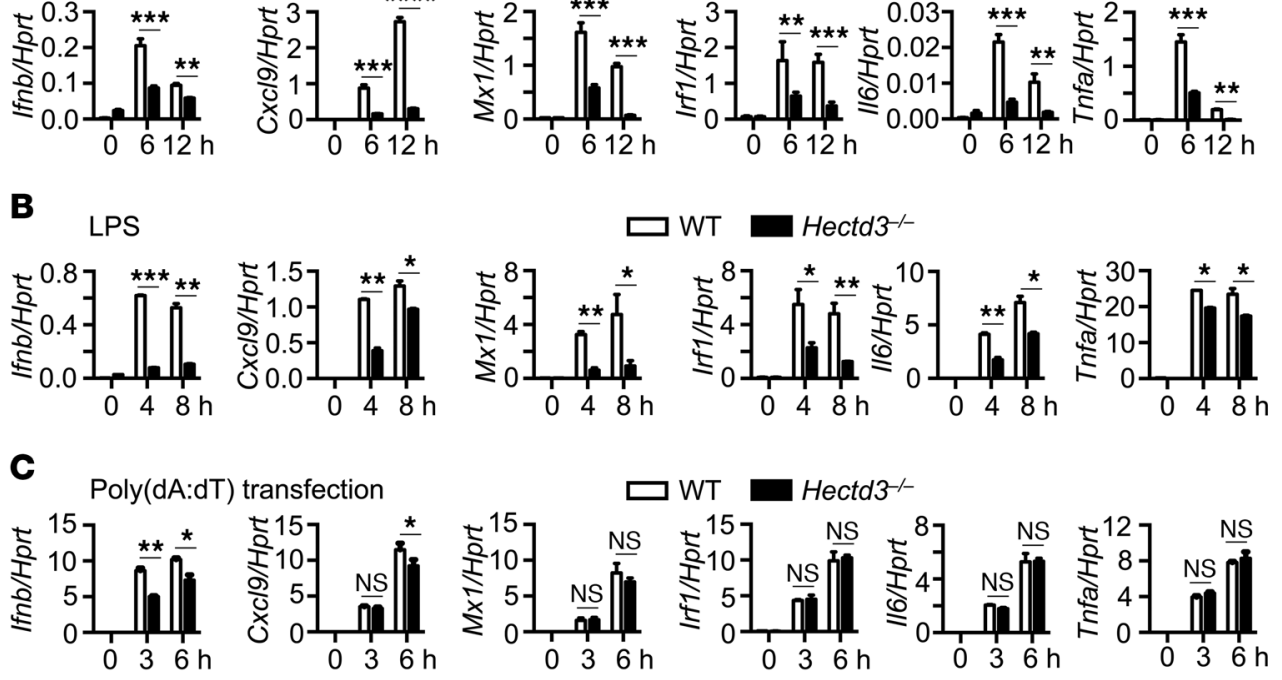
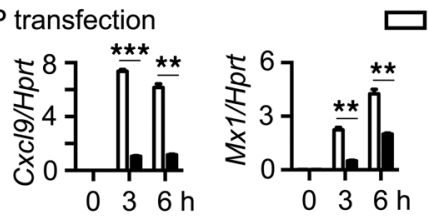
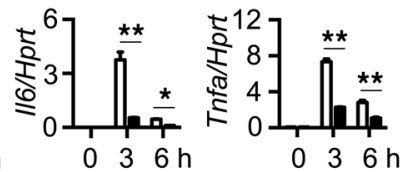

Figure 5. HECTD3 deficiency inhibits TRIF- and STING-dependent induction of type I IFN. (A) Gene expression analysis of WT and Hectd3 $3^{-/-}$ BMDMs in response to poly $(\mathrm{I}: \mathrm{C})$ stimulation for indicated times. (B) Gene expression analysis of WT and Hectd $3^{-/-}$BMDMs in response to LPS stimulation for indicated times. (C) Gene expression analysis of WT and Hectd $3^{-/}$BMDMs in response to poly $(\mathrm{dA}: \mathrm{dT}$ ) transfection treatment for indicated times. (D) Gene expression analysis of WT and Hectd $3^{-/-}$ BMDMs in response to $2^{\prime}{ }^{\prime}$-cGAMP transfection treatment for indicated times. Data represent 3 independent experiments and are presented as mean \pm SEM. ${ }^{*} P<0.05$; ${ }^{* *} P<0.01$; ${ }^{* * *} P<0.001 ;{ }^{* * *} P<0.0001$. not $\mathrm{Hectd}^{-/-}$BMDMs (Supplemental Figure 4A and Supplemental Table 1). After a 12-hour infection with $F$. novicida, 1,115 and 1,109 genes were upregulated in WT and Hectd $3^{-/-}$BMDMs, respectively (Supplemental Figure 4, D and E, and Supplemental Table 1), and 142 genes had higher expression in WT BMDMs than in Hectd3-BMDMs (Supplemental Figure 4A and Supplemental Table 1). The gene set with lower expression in Hectd $3^{-/-}$BMDMs than in WT BMDMs mostly comprised Ifnb and its inducible genes, including Cxcl10, Irf1, Irf7, Mx1, Mx2, Gbp, Ifit, and those encoding Irgb family members (Figure 4A). The remarkably decreased expression of Ifnb and its inducible genes Cxcl9, MxI, Irf1, Isg15, and Ifit1 in response to $F$. novicida infection was confirmed by quantitative reverse-transcriptase PCR (qRT-PCR) analysis of BMDMs (Figure $4 \mathrm{~B}$ and Supplemental Figure $5 \mathrm{~A}$ ). Impaired IFN- $\beta$ production in $\mathrm{Hectd}^{-/-}$BMDMs after F. novicida infection was confirmed by ELISA (Figure 4C). Consistent with these findings, levels of activated TBK1 and IRF3, which are critical for type I IFN production and type I IFN-activated molecules STAT1 and STAT3, were substantially inhibited in the absence of an HECTD3 response to $F$. novicida infection (Figure 4D). In contrast, I $\mathrm{KB} \alpha, \mathrm{IKK} \alpha, \mathrm{ERK}, \mathrm{P} 38$, and JNK activation was not differentially regulated in F. novicidainfected WT and Hectd3 ${ }^{-/-}$BMDMs (Figure 4D). Expression of Ifnb and its inducible genes was also lower in $\mathrm{Hectd3}^{-/-} \mathrm{BMDMs}$ than in WT BMDMs after BCG-GFP, L. monocytogenes, E. coli, and even DNA virus HSV infection (Supplemental Figure 5, B-D, and Supplemental Figure 6, A and B).

F. novicida infection in macrophages is detected by the DNA-sensing AIM2 inflammasome, and type I IFN is essential for cytosolic bacteriolysis and release of bacterial DNA $(38,39)$. As predicted, activation of the AIM2 inflammasome, indicated by caspase- 1 maturation, subsequent release of substrate IL-1 $\beta$, and $\mathrm{LDH}$ release during $F$. novicida infection, was substantially inhibited in Hectd3 $3^{-/-}$BMDMs (Figure 4C and Supplemental Figure 7, A and B). However, HECTD3 was not required to activate LPS/ATP-induced NLRP3 inflammasome, Salmonella-induced NLRC4 inflammasome, or DNA-induced AIM2 inflammasome (Supplemental Figure 8, A-C). This finding suggests that HECTD activated the AIM2 inflammasome induced by F. novicida infection by mediating type I IFN production. Moreover, cell death and associated activation of caspase- 3 and -8 , expression of ZBP1 and caspase-11, and phosphorylation of MLKL were reduced in Hectd $^{-/-}$BMDMs after F. novicida infection (Supplemental Figure 7, A and C). Levels of autophagy, determined by LC3-II conversion and lysosomal biogenesis, as indicated by TFEB expression, were comparable between F. novicida-infected WT and Hectd3 $3^{-1-}$ BMDMs (Supplemental Figure 8D), indicating that reduced cell death in $\mathrm{Hectd}^{-/-}$BMDMs was not a feedback from autophagy and lysosomal biogenesis (40). Similarly, caspase-1 activation triggered by BCG-GFP or L. monocytogenes infection was substantially lower in Hectd $3^{-/-}$BMDMs than in WT BMDMs (Supplemental Figure $8 \mathrm{E}$ ). These findings indicate that HECTD3 positively controls type I IFN production, which leads to activation of downstream molecules and cell death triggered by $F$. novicida.

HECTD3 mediates TRIF- and STING-dependent induction of type I IFN. Multiple E3 ubiquitin ligases play essential roles in type I IFN production by mediating the ubiquitination of key regulators of Ifnb expression. To identify the molecules targeted by HECTD3, we treated WT and Hectd3 $3^{-/-}$BMDMs with ligands specific for activating various pathogen-recognition receptors. In the case of TLR engagement, activation of WT and Hectd $3^{-/-}$BMDMs in response 
A

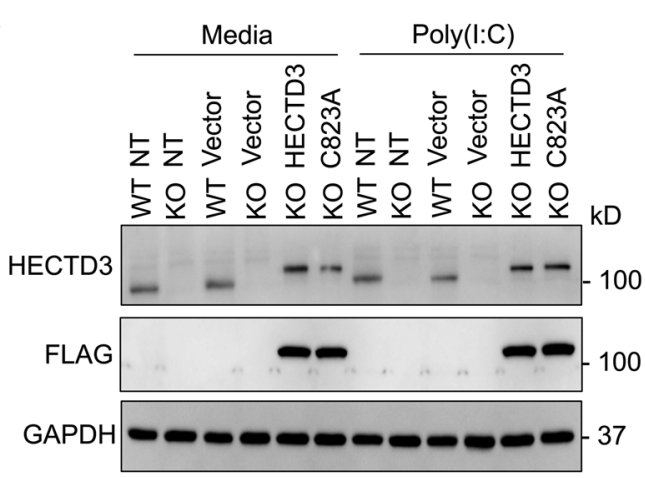

B
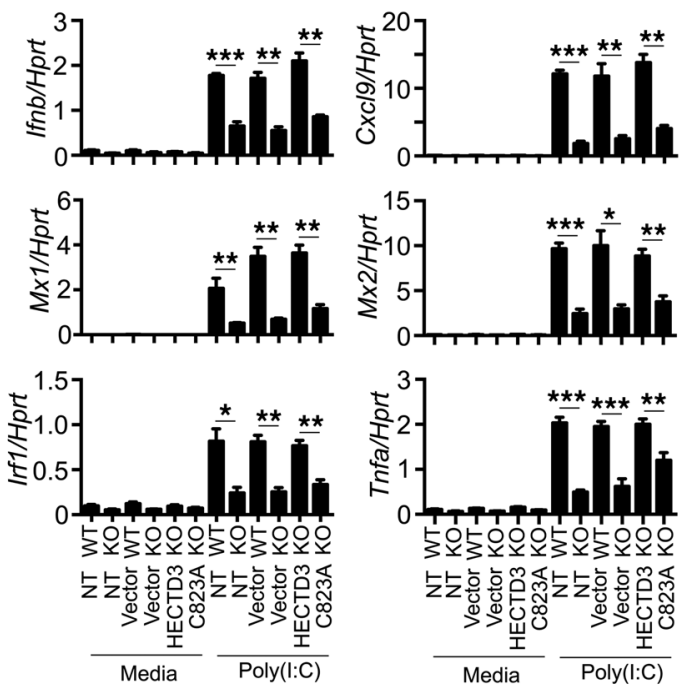

Figure 6. HECTD3 transfection rescues the activation of type I IFN signaling in Hectd3 $^{-/-}$cells. (A) Immunoblot analysis of HECTD3 and FLAG in WT and Hectd3 $^{-/-}$MEFs transfected with the vector control and FLAG-tagged WT HECTD3 or mutant HECTD3 C823A plasmids as indicated. (B) Gene expression analysis of WT and Hectd3 $3^{-/-}$MEFs transfected with indicated plasmids in response to poly $(\mathrm{I}: \mathrm{C})$ stimulation. Data represent 3 independent experiments and are presented as mean \pm SEM. ${ }^{*} P<0.05 ;{ }^{* *} P<0.01 ;{ }^{* *} P<0.001$.

to ligands TLR2, TLR7, or TLR9 was comparable (Supplemental Figure 9, A and B). Interestingly, HECTD3 deficiency led to considerably reduced expression of Ifnb and its inducible genes after TLR3 or TLR4 engagement with their respective agonists (Figure 5, A and B).

The cytosolic DNA sensor cGAS is required for STINGdependent type I IFN response to F. novicida infection (41). To examine the role of HECTD3 in activating the cGAS/STING axis, we treated WT and Hectd3 $3^{-/}$BMDMs with cGAS agonist poly(dA:dT) and STING ligand 2'3'-cGAMP. HECTD3 deficiency led to defective Ifnb expression in response to poly(dA:dT) stimulation (Figure 5C). Notably, the expression of Ifnb and its inducible genes $\mathrm{Cxcl9}, \mathrm{M} x 1$, and $\operatorname{Irf1}$ and proinflammatory cytokine genes Il6 and Tnfa was reduced in Hectd3 $3^{-/}$BMDMs after 2'3'-cGAMP stimulation (Figure 5D). However, the activation of cytosolic RNA sensors RIG-I and MDA-5 engaged with dsRNAs or polyinosinic:polycytidylic acid (poly[I:C]) was not affected in the absence of HECTD3 in BMDMs or mouse embryonic fibroblasts (MEFs) (Supplemental Figure 9, C and D). To further confirm the critical role of HECTD3 in type I IFN production, we performed an HECTD3 rescue experiment in $\mathrm{Hectd3}^{-/}$MEFs. Transfected WT HECTD3, but not the mutant HECTD3-C823A (described below), in $\mathrm{Hectd3}^{-/}$MEFs restored the decreased expression of Infb, Cxcl9, Mx1, Mx2, Irf1, and Tnfa induced by poly(I:C) treatment (Figure 6, A and B). These results indicate that HECTD3 plays a key role in regulating TRIF- and STING-dependent type I IFN production.

HECTD 3 targets TRAF3. TRAF3 is reported to mediate TRIFdependent and -independent type I IFN production (42), and TBK1 activation induced by STING engagement might occur in association with TRAF3 $(29,43)$. Thus, HECTD3 might regulate TRIF- and STING-dependent signaling by mediating TRAF3 activation. To determine whether HECTD3 interacts with TRAF3, we transiently expressed HECTD3 or HA-tagged HECTD3 with FLAG-tagged TRAF3 in HEK293T cells. IP of FLAG or HA revealed an interaction between HECTD3 and TRAF3 (Figure 7A). To determine whether HECTD3 regulates TRAF3 activation through its E3 ligase activity, we analyzed HECTD3mediated polyubiquitination of TRAF3. In the presence of HECTD3, TRAF3 polyubiquitination was remarkably increased, indicating that HECTD3 directly interacted with TRAF3 and was required for TRAF3 polyubiquitination (Figure 7B). To pinpoint the HECTD3 domain essential for TRAF3 polyubiquitination, we generated the HECTD3 mutant HECTD3-C823A, in which the conserved cysteine residue at position 823 within the HECT domain was replaced with alanine, and analyzed polyubiquitination. Notably, the level of TRAF3 polyubiquitination in the presence of HECTD3-C823A was much lower than that of WT HECTD3 (Figure 7C), indicating that the catalytic domain of HECTD3 was required for it to polyubiquitinate TRAF3. TRAF3 has been established as a critical linker between adaptors and the downstream regulatory kinase TBK1 (42). Consistent with this, we found that HECTD3 interacts with TBK1 and upstream adaptors TRIF and STING (Supplemental Figure 10).

To confirm the interaction between endogenous HECTD3 and TRAF3, we performed co-IP experiments with HECTD3 and TRAF3 in WT and Hectd3 ${ }^{--}$BMDMs. Strikingly, HECTD3 was immunoprecipitated by TRAF3 in WT BMDMs, but not in Hectd $3^{-1-}$ BMDMs (Figure 7D). The TRAF3-TBK1 interaction induced by $F$. novicida infection was impaired in the absence of HECTD3 (Figure 7D). Confocal microscopy analysis revealed that colocalization of TRAF3 and TBK1 in response to F. novicida infection was lower in Hectd3 $^{-/-}$BMDMs than in WT BMDMs, and TBK1 phosphorylation induced by F. novicida infection was impaired in $\mathrm{Hectd}^{-/-} \mathrm{BMDMs}$ (Figure 7, E and F). In addition, the ubiquitination of endogenous TRAF3 induced by $F$. novicida infection was reduced in the absence of HECTD3 (Figure 7G). Thus, these results indicate that HECTD3 targets TRAF3 to mediate its polyubiquitination.

HECTD3 promotes type I IFN signaling by catalyzing K63-linked polyubiquitination of TRAF3. To identify the type of TRAF3 polyubiquitination mediated by HECTD3, we cotransfected HEK293T cells with HECTD3, TRAF3, and HA-tagged WT ubiquitin and K48-, K63-, K6-, K11-, K27-, K29-, or K33-specific ubiquitin. HECTD3 promoted polyubiquitination of TRAF3 in the presence of HA-tagged WT ubiquitin and HA-tagged K63-specific ubiquitin, but not of HA-tagged other linkage-specific ubiquitin (Figure 
A

FLAG-TRAF3 - + pCMV-Vector +

HA-HECTD3 - + pCDH-Vector +

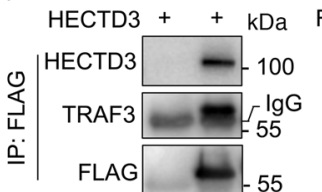
LAG-TRAF3 $++\mathrm{kDa}$

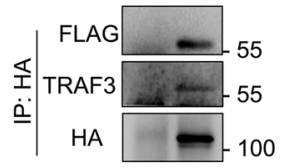

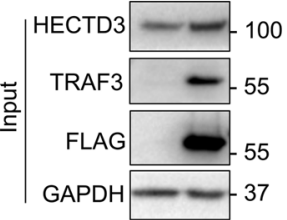

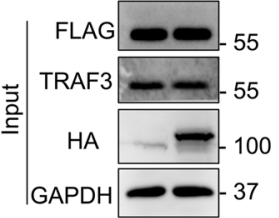

E

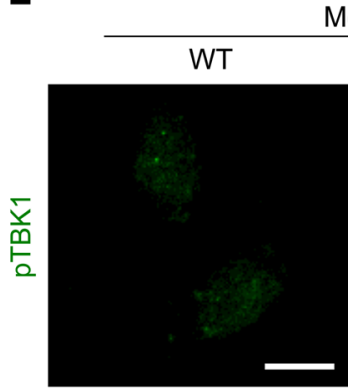

Media $\mathrm{Hectd}^{-}-$
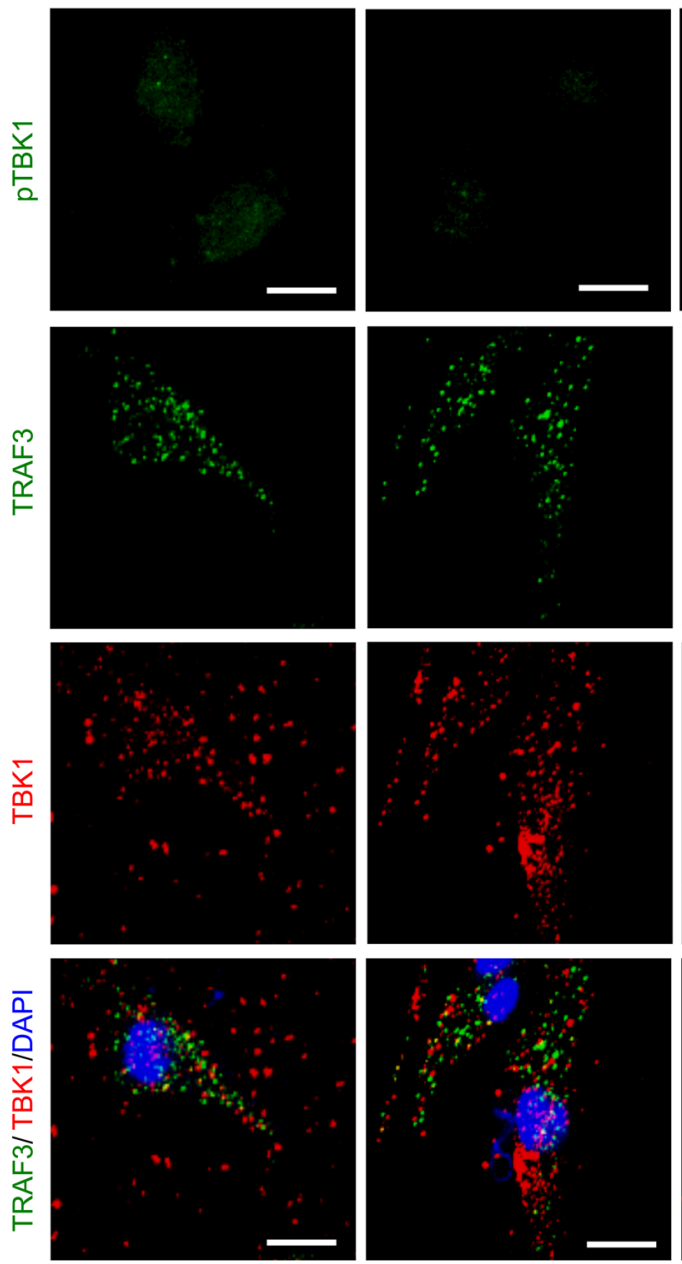

B

$\mathrm{HA}-\mathrm{Ub}+-++$

F-TRAF3 - + + +

Vector - - + -

HECTD3 - - - + kDa
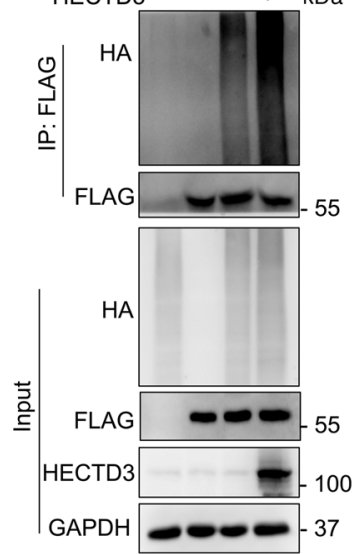

C

F-TRAF3 ++++

HA-Ub - + +

Vector - + -

HECTD3(C823A) - - + $+\mathrm{kDa}$
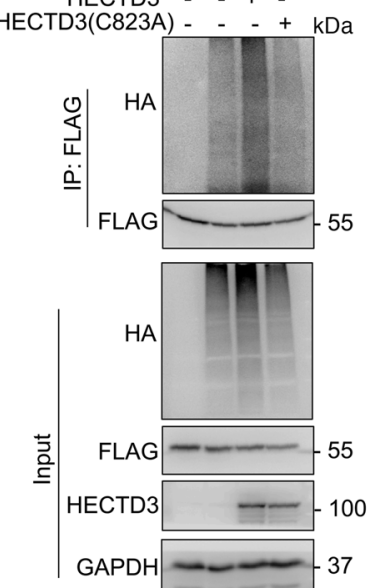

D Med F.n.

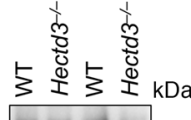

HECTD3 100

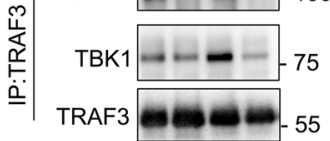

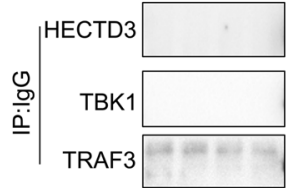

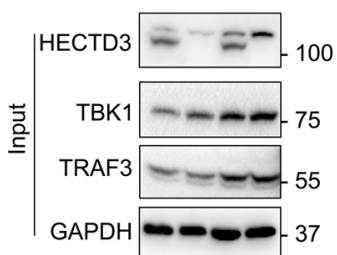

$\mathbf{F}$

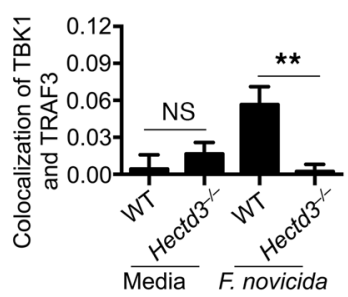

G

Media F. novicida
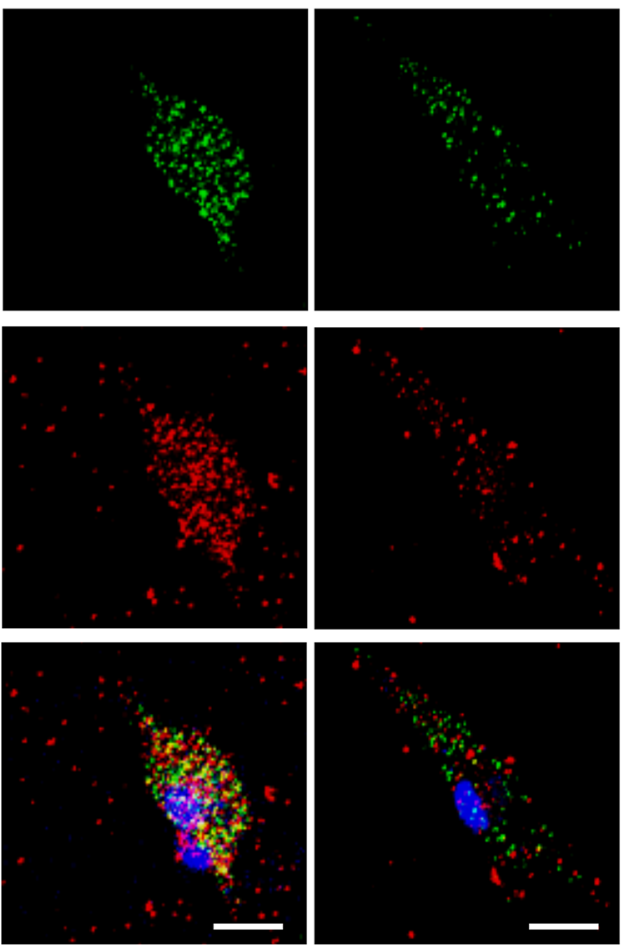

々 突 $5 \frac{\text { के }}{0}$ $\mathrm{kDa}$

芯

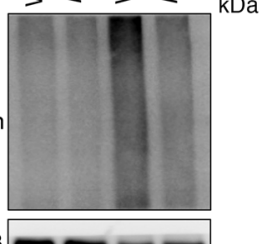

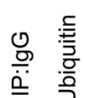

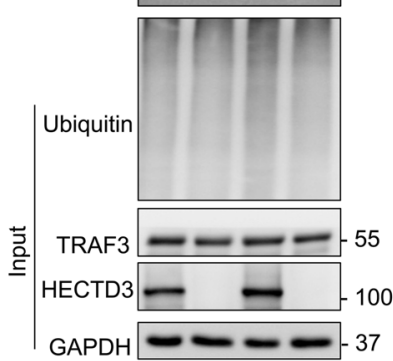


Figure 7. HECTD3 interacts with the TRAF3-TBK1 complex. (A) Immunoblot analysis of HECTD3 and TRAF3 that coimmunoprecipitated with FLAG-TRAF3 or HA-HECTD3 from lysates of HEK293T cells transfected with plasmids, as indicated. (B) Co-IP analysis of polyubiquitination of TRAF3 mediated by HECTD3 in HEK293T cells transfected with plasmids as indicated. (C) Co-IP analysis of polyubiquitination of TRAF3 by WT and mutant HECTD3 (C823A) in HEK293T cells transfected with plasmids as indicated. (D) Co-IP analysis of endogenous TRAF3 with TBK1 and HECTD3 in WT and Hectd3 $3^{-/}$BMDMs with or without $F$. novicida infection for 6 hours. (E) Confocal microscopy analysis of colocalization of TBK1 and TRAF3, and phosphorylation of TBK1 cellular localization in WT and

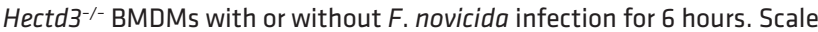
bars: $10 \mu \mathrm{m}$. (F) Quantification of colocalization of TBK1 and TRAF3 in $\mathbf{E}$ ${ }^{*} P<0.01$. (G) Co-IP analysis of polyubiquitination of endogenous TRAF3

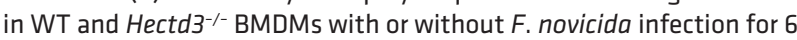
hours. Data represent 3 independent experiments for $\mathbf{A}-\mathbf{F}$ and 2 independent experiments for $\mathbf{G}$.

8A and Supplemental Figure 11A). However, HECTD3 did not catalyze TRAF3 polyubiquitination in the presence of mutant ubiquitin-K63R, which harbored a lysine-to-arginine substitution at position 63 (Figure 8B). These results indicate that HECTD3 mediates K63-linked polyubiquitination of TRAF3 and promotes type I IFN production, which is consistent with the fact that K63linked polyubiquitination activates various signaling pathways in the innate immune response (44).

TRAF3 harbors multiple lysine residues within the N-terminal RING finger and zinc finger domains and the C-terminal TRAF domain. We first studied lysine residues within the $\mathrm{N}$-terminal domain that are crucial for recruiting E3 ubiquitin ligases and ubiquitination events $(45,46)$. To identify the lysine residues in HECTD3mediated K63-linked polyubiquitination of TRAF3, we generated 5 TRAF3 mutants (K138R, K154R, K156R, K160R, and K168R) in which lysine residues were replaced with arginine residues at positions 138, 154, 156, 160, and 168, respectively. Notably, K63-linked polyubiquitination of TRAF3 was diminished in K138R, but not other mutants (Figure 8C). Our results indicate that HECTD3 promotes K63-linked polyubiquitination of TRAF3 at Lys138.

To determine whether HECTD3-mediated K63-linked polyubiquitination of TRAF3 at K138 is essential for type I IFN production, we transfected WT and mutant TRAF3 (K138R, K160R, or K168R) into Traf3 $3^{--}$MEFs and assessed Ifnb expression under HECTD3 cotransfection. The expression of Cxcl9, Mx1, and Irf1 was significantly higher in Traf3 ${ }^{-/-}$MEFs cotransfected with WT TRAF3 and HECTD3 than in those transfected with either WT TRAF3 or HECTD3 alone in response to poly(I:C) stimulation (Figure 8D). Notably, transfection with WT and mutant TRAF3 K160R or K168R, but not with mutant TRAF3 K138R, rescued the expression of Ifnb, Cxcl9, Mx1, Mx2, Irf1, and Tnfa in the presence of HECTD3 (Figure 8D). These findings demonstrate that HECTD3 regulates type I IFN by mediating K63-linked polyubiquitination of TRAF3 at K138 during bacterial infection (Supplemental Figure 11B).

\section{Discussion}

Type I IFN production triggered by the recognition of PRRs is an essential step in the innate immune response to invading viruses. However, how type I IFN in the host counteracts infections by intracellular bacteria remains less clear. Here, we show that
HECTD3 functions as a negative regulator in host defense against infections by intracellular bacteria by promoting K63-linked polyubiquitination of TRAF3, which promotes type I IFN production. Further, HECTD3 plays a detrimental role in host defense against intracellular bacteria by facilitating the dissemination of bacteriacarrying leukocytes.

Type I IFN is detrimental for host defense against intracellular bacteria, especially those lacking classical virulence factors, such as Francisella, Mycobacterium, and Listeria (3). However, the mechanism by which type I IFN attenuates host defense remains obscure. Two putative mechanisms have been proposed: (a) early type I IFN inhibits IL-17 production by $\gamma \delta$ T cells, which dampens neutrophil migration (47), and (b) immune cells triggered by type I IFN serve as a replication niche for infection by intracellular bacteria (37). We found the number of circulating bacteriacontaining leukocytes in the bloodstream was much lower in $\mathrm{Hectd}^{-/-}$mice than in WT mice, indicating that infected immune cells serve as a replication niche and carrier to disseminate intracellular bacteria. Our study reiterates that type I IFN plays a detrimental role in host defense against intracellular bacteria by tendentiously providing an intracellular replication niche for bacteria. In addition, impaired type I IFN production reduces cell death and inflammasome activation in Hectd3 $3^{-/}$BMDMs, which might further limit the spread of bacterial infection.

TRAF3 acts as an essential scaffold protein to coordinate the assembly of signaling complexes downstream of PRRs and their adaptor proteins to promote IRF3 phosphorylation by TBK1 and negatively regulate the activation of noncanonical NF- $\kappa \mathrm{B}$ and mitogen-activated protein kinase $(42,45,48)$. Increasing evidence supports that protein ubiquitination mediated by E3 ubiquitin ligases is key in TRAF-dependent signaling and innate immunity (49). TRAF3 activity can be mediated through several types of ubiquitination. In particular, cIAP1/2- and TRAF6-mediated K48-linked polyubiquitination of TRAF3 results in its proteasomal degradation and induction of proinflammatory cytokines, whereas TRIF-mediated K63-linked polyubiquitination of TRAF3 activates IRF3 and type I IFN response (45). Recently, K33-linked polyubiquitination of TRAF3 was shown to modulate the expulsion of intracellular bacteria, independently of type I IFN production (46). In addition, TRAF3 contains an N-terminal RING domain and might be capable of autoubiquitination, but this has not been confirmed for endogenous TRAF3 under physiological conditions (48). TRAF3 is a versatile regulator, and its biological effect is highly dependent on context and mediated by distinct types of ubiquitination. Unlike K48-linked polyubiquitination, K63-linked polyubiquitination of TRAF3 provides binding sites for interacting proteins involved in the signaling activation pathway. Our study demonstrates that HECTD3-mediated K63-linked polyubiquitination of TRAF3 is essential for TRAF3-TBK1 interaction and TBK1 phosphorylation. HECTD3 also stabilizes the TRAF3-TBK1 complex, which is required for TRIF- and STING-dependent induction of type I IFN. This is consistent with the observations that K63-linked polyubiquitination of TRAF3 is dependent on endocytosis and the TRIF adaptor and that TRAF3 is associated with STING in the cytosolic DNA-triggered activation of signaling $(29,45)$. The independent role of HECTD3 in MAVS-mediated type I IFN production suggests that TRAF3 is dispensable for RIG-I/MAVS axis signaling, which is 
A
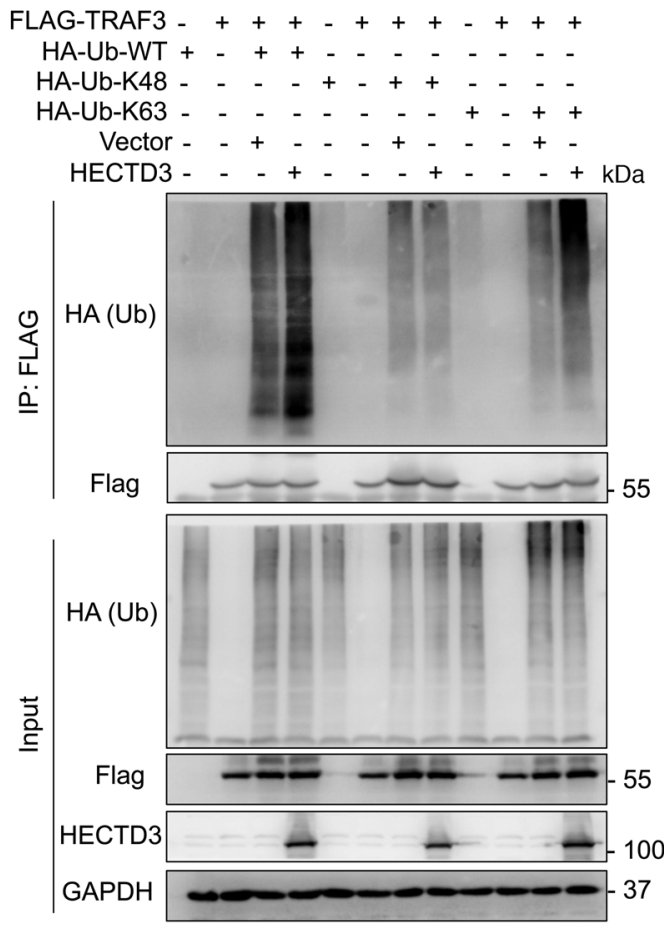

B
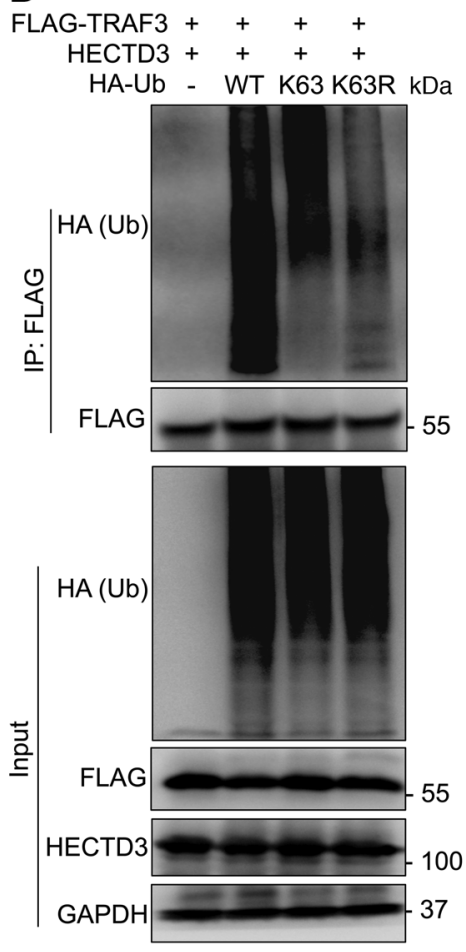

C
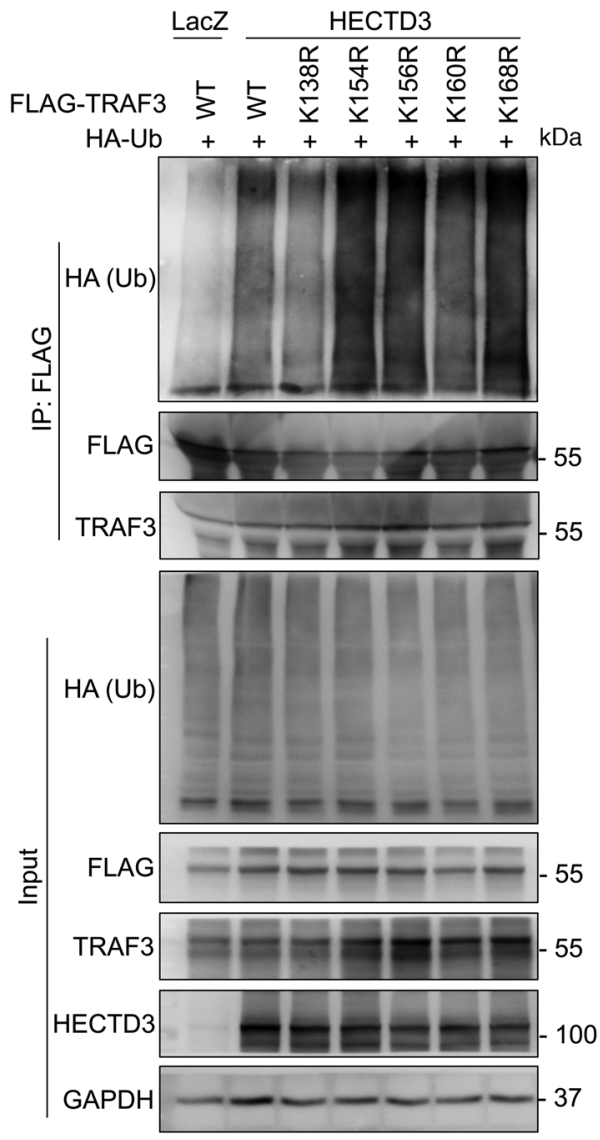

D

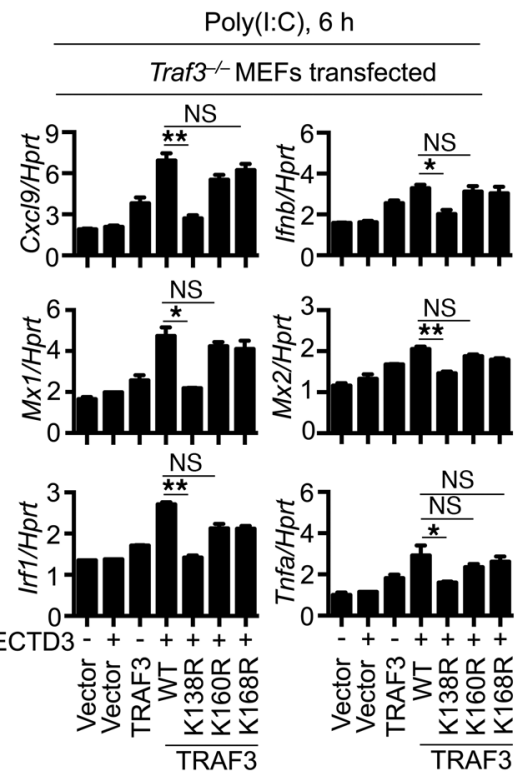

Figure 8. HECTD3-mediated K63linked polyubiquitination of TRAF3 and its function. (A) Co-IP analysis of K48- or K63-linked polyubiquitination of TRAF3 mediated by HECTD3 in HEK293T cells transfected with plasmids as indicated. (B) Co-IP analysis of WT K63- and mutant K63-linked (K63R-linked) polyubiquitination of TRAF3 mediated by HECTD3 in HEK293T cells transfected with plasmids as indicated. (C) Co-IP analysis of K63-linked polyubiquitination of WT TRAF3 and its mutants K138R, K154R, K156R, K160R, and K168R by HECTD3 in HEK293T cells transfected with plasmids, as indicated. (D) Gene expression analysis of If $n b, C \times C \mid 9, M \times 1, M \times 2$, Irf1, and Tnfa in Traf3 $3^{-/}$MEFs transfected with HECTD3 combined with WT or mutant TRAF3 plasmids (K138R, K160R, and K168R), as indicated by the lentiviral transduction system. Data represent 3 independent experiments and are presented as mean \pm SEM. ${ }^{*} P<0.05 ;{ }^{* *} P<0.01$. consistent with the finding that TRAF2, -5 , and -6 are essential for MAVS signaling and antiviral immune responses $(49,50)$. Intracellular bacteria primarily use the cGAS/STING axis for type I IFN production, whereas extracellular bacteria use the TLR4-TRIF pathway for type I IFN induction (3). Whether HECTD3 activity is modulated by bacterial pathogens, what the function of HECTD3mediated TRAF3 activation is in host defense against infection by
DNA and RNA viruses, and whether Hectd $3^{-/-}$mice have roles independent of type I IFN in host defense against infection by intracellular bacteria need further examination.

Despite the fact that K63-linked polyubiquitination of TRAF3 was recently documented by RNF166, Mint3, and NEDD4 (5153), our study provides comprehensive evidence of E3 ubiquitin ligase mediating TRAF3 K63-linked polyubiquitination to regu- 
late the type I IFN response during bacterial infection. In addition, negative regulation of host defense by HECTD3-mediated K63-linked polyubiquitination of TRAF3 supports the idea that infection by intracellular bacteria can be inhibited by interfering with its activity. Thus, we reveal a previously unrecognized functional role for HECTD3 in negatively regulating host defense against infections by intracellular bacteria and dissect the mechanism by which HECTD3 regulates type I IFN responses. Our results provide the grounds for exploring HECTD3 as a potential drug target for controlling infections by intracellular bacteria and inflammatory diseases.

\section{Methods}

Mice. Hectd3 $3^{-/-}$mice were generated by Taconic Farms Inc. (TF2706, Taconic Knockout Repository). Exons 10 through 17 of the HECTC3encoding gene were replaced with a fragment containing the selection marker for puromycin resistance, which resulted in deletion of functional HECT domain. The strategy for construction of the targeting vector is illustrated in Supplemental Figure 1A. Embryonic stem cells from the $129 / \mathrm{SvEv}$ strain with a gene deletion in Hectd 3 were microinjected into C57BL/ 6 blastocysts. Chimeric offspring were backcrossed to FVB mice for 11 generations. Hectd3-knockout mice were confirmed by qRT-PCR and Western blot analysis (Supplemental Figure 1, B and C). All mice were kept in specific pathogen-free conditions within the Animal Resource Center at the Kunming Institute of Zoology.

Bacterial culture and infection of mice. The bacterial strains used in this study, including F. novicida strain U112, GFP-expressing $F$. novicida, L. monocytogenes, Salmonella Typhimurium, and E. coli, were grown as previously described (40). GFP-expressing $M$. bovis BCG was grown with gentle agitation (100 rpm) in Middlebrook 7H9 medium supplemented with $0.05 \%$ Tween 80 and $10 \%$ oleic acid-albumindextrose-catalase complex (OADC).

Eight- to ten-week-old Hectd $3^{-/-}$mice and sex-matched littermate WT controls were infected subcutaneously with $F$. novicida $\mathrm{U} 112\left(3.0 \times 10^{5} \mathrm{CFUs}\right.$ per mouse), intraperitoneally with L. monocytogenes $\left(6.0 \times 10^{4} \mathrm{CFUs}\right.$ per mouse $)$ or E. coli $\left(1.0 \times 10^{8} \mathrm{CFUs}\right.$ per mouse), and intranasally with GFP-expressing BCG $\left(7.5 \times 10^{6}\right.$ CFUs per mouse) or GFP-expressing $F$.novicida (5,000 CFUs per mouse), as indicated. Mice were weighted and monitored daily over time. Mice were euthanized at indicated days after infection, and liver, spleen, and lung were harvested to determine the bacterial burden as described previously (40). For immune cell-infiltration analysis, whole blood and lung from WT and Hectd $3^{-/-}$mice were analyzed by flow cytometry analysis.

Bacterial infection of macrophages. To generate BMDMs, BM cells were cultured in L929 cell-conditioned DMEM/F-12 supplemented with $10 \%$ FBS, $1 \%$ nonessential amino acids, and $1 \%$ penicillin-streptomycin for 5 days. BMDMs were seeded in 12-well plates (1 million cells per well) and cultured overnight. The next day, cells were washed and supplied with fresh media without antibiotics. BMDMs were infected with bacteria for the indicated times. BMDMs were lysed in radioimmunoprecipitation assay (RIPA) buffer with protease inhibitor and phosphatase inhibitors (Roche) for immunoblot analysis.

Bacterial killing assay. BMDMs were infected with $F$. novicida with an MOI of 10 for 2 hours and washed; gentamicin $(50 \mu \mathrm{g} / \mathrm{ml})$ was added to kill extracellular bacteria. After 1 hour, cells were washed twice and cultured in fresh media. BMDMs were lysed in PBS at indicated times after infection, serially diluted, plated onto TSB agar plates, and incubated overnight for CFU enumeration.

Preparation of tissue sample for histopathologic staining and immunoblot analysis. Liver and spleen tissues were fixed in 10\% formalin and embedded in paraffin and sectioned ( $5 \mu \mathrm{m}$ thickness). Sections were stained with H\&E and examined. For immunochemical staining, tissue sections were stained with anti-Ly-6G antibody, followed by incubation for 30 minutes with secondary antibody. Tissues were homogenized in RIPA buffer with protease and phosphatase inhibitors for immunoblot analysis. Protein concentration was determined using the BCA Kit (Pierce) according to the manufacturer's instructions.

Immunoblot analysis and antibodies. Samples were separated by $12 \%$ SDS-PAGE, followed by electrophoretic transfer onto PVDF membranes. Samples were then blocked with 5\% nonfat milk and further incubated overnight in primary antibody at $4^{\circ} \mathrm{C}$ as described previously (40). The following primary antibodies were used: anti-IкB $\alpha$ (Cell Signaling Technology, catalog 9242),

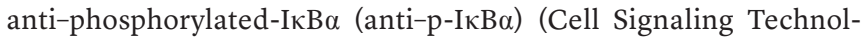
ogy, catalog 2859), anti-ERK (Santa Cruz Biotechnology Inc., catalog sc-94), anti-p-ERK (Santa Cruz Biotechnology Inc., catalog sc-7383), anti-caspase-1 (Adipogen, catalog AG-20B-0042), antiLC3B (Novus Biologicals, catalog NB600-1384), anti-caspase-3 (Cell Signaling Technology, catalog 9661 and 9662), anti-TFEB (Bethyl Laboratories, catalog A303-673A), anti-p-TBK1 (Cell Signaling Technology, catalog 5483), anti-TBK1 (Cell Signaling Technology, catalog 3504), anti-p-IRF3 (Cell Signaling Technology, catalog 4947), anti-IRF3 (Cell Signaling Technology, catalog 4302), anti-caspase-8 (Cell Signaling Technology, catalog 8592), anti-pSTAT1 (Cell Signaling Technology, catalog 7649), anti-STAT1 (Cell Signaling Technology, catalog 14994s), anti-p-STAT3 (Cell Signaling Technology, catalog 9131), anti-STAT3 (Cell Signaling Technology, catalog 9132), anti-p-MLKL (Abcam, catalog ab196436), anti-MLKL (Abgent, catalog ap14272b), anti-caspase-11 (Novus Biologicals, catalog NB120-10454), anti-ZBP1 (Adipogen, catalog AG-20B-0010), anti-p-IKK $\alpha / \beta$ (Cell Signaling Technology, catalog 2697s), anti-IKK $\alpha$ (Cell Signaling Technology, catalog 2682), antip-JNK (Cell Signaling Technology, catalog 9251s), anti-JNk (Cell Signaling Technology, catalog 9252), anti-p-P38 (Cell Signaling Technology, catalog 9211s), anti-P38 (Cell Signaling Technology, catalog 9212s), anti-STING (Cell Signaling Technology, catalog 13647s), anti-TRAF3 (Abcam, ab36988), anti-TRAF6 (Santa Cruz Biotechnology Inc., catalog sc-8409), anti-HA (Santa Cruz Biotechnology Inc., catalog sc-805), anti-FLAG (MilliporeSigma, cata$\log$ F3165), anti-TRIF (BioLegend, catalog 657102), anti-ubiquitin (MilliporeSigma, catalog 04-263), and anti-GAPDH (Cell Signaling Technology, catalog 5174). Anti-HECTD3 antibody was previously described (35). HRP-labeled anti-rabbit, anti-mouse, or anti-goat (Cell Signaling Technology) was used as the secondary antibody.

Immunofluorescence staining and microscopy. For TRAF3, TBK1, and p-TBK1 immunostaining, infected and uninfected BMDMs were fixed in $4 \%$ paraformaldehyde for 15 minutes at room temperature. Cells were washed with PBS and blocked in 1× ELISA buffer with 0.1\% saponin for 1 hour. Cells were stained with anti-TRAF3 (Abcam, ab36988), anti-TBK1 (Novus Biologicals, 108A429), or anti-p-TBK1 (Cell Signaling Technology, 5483), all at 1:300 dilution, overnight at $4^{\circ} \mathrm{C}$. Cells were washed, stained with fluorescence-conjugated secondary antibody for 40 minutes at $37^{\circ} \mathrm{C}$, and mounted using mounting 
medium (Vector Laboratories, H-1200). Cells were observed on the Olympus FluoView 1000 confocal microscope (Olympus) for image acquisition and data analysis.

Flow cytometry analysis. For flow cytometric analysis of $\mathrm{T}$ cells, B cells, neutrophils, macrophages, and dendritic cells, cells prepared from lungs and peripheral blood were stained using a subset of antibodies. Cell preparation and staining were carried out as described previously $(54,55)$, and cells were analyzed on a BD LSRFortessa Cell Analyzer (BD Biosciences). Dead cells were excluded by propidium iodide (PI) staining.

Real-time quantitative PCR. Total RNA was isolated from tissues or cells using TRIzol (Invitrogen) and purified by RNeasy Mini Kit (QIAGEN), cDNA was reverse transcribed using Superscript III (Invitrogen). Real-time quantitative PCR (qPCR) was performed on the BioRad CFX-96 Touch Real-Time Detection System. Primer sequences are listed in Supplemental Table 2.

RNA sequencing and data analysis. Total RNA was extracted from F. novicida-infected and uninfected WT and Hectd3 $3^{-/}$BMDMs and subjected to commercial RNA-sequencing (RNA-seq) analysis (Novogene). A total of $3 \mu \mathrm{g}$ RNA per sample was used as input material for the RNA sample preparations. Sequencing libraries were generated using NEBNext Ultra RNA Library Prep Kit for Illumina following the manufacturer's recommendations, and index codes were added to attribute sequences to each sample. The clustering of the index-coded samples was performed on a cBot Cluster Generation System using TruSeq PE Cluster Kit v3-cBot-HS (Illumina) according to the manufacturer's instructions. After cluster generation, the library preparations were sequenced on an Illumina HiSeq platform, and $125 \mathrm{bp} / 150$ bp paired-end reads were generated.

The clean RNA-seq data were aligned to the reference genome using TopHat v2.0.12. HTSeq v0.6.1 was used to count the reads numbers mapped to each gene. Afterwards, fragments per kilobase of exon per million mapped fragments (FPKM) of each gene were calculated based on the length of the gene and read counts mapped to this gene. Prior to differential gene expression analysis, for each sequenced library, the read counts were adjusted by the edgeR program package through 1 scaling normalized factor. Differential expression analysis of 2 conditions was performed using the DEGSeq R package (1.20.0). $P$ values were adjusted using the Benjamini-Hochberg method. Corrected $P$ values of 0.005 and $\log _{2}$ (fold changes) of 1 were set as the threshold for significantly differential expression. Heatmaps were generated to show the normalized gene expression among the samples by using Package pheatmap software.

Plasmidstransfection and co-IP. The plasmids expressing HECTD3, TBK1, TRIF, and STING were previously described (18, 20, 35). TRAF3 was amplified from a cDNA library and cloned into PCMV-FLAG vector. Site-directed mutations were generated using the QuikChange Site-Directed Mutagenesis Kit. All plasmids were confirmed by DNA sequencing. Lipofectamine 2000 reagents (Invitrogen) were used for transient transfection of plasmids into HEK293T cells.

For IP, whole HEK293T cells collected 36 hours after transfection and WT and Hectd $3^{-/-}$BMDMs with or without $F$. novicida infection were lysed in IP buffer composed of $1.0 \%$ (vol/vol) NP-40, 50 mM Tris- $\mathrm{HCl}, \mathrm{pH}$ 7.4, $50 \mathrm{mM}$ EDTA, $150 \mathrm{mM} \mathrm{NaCl}$, and protease/ phosphatase inhibitor cocktails (Biotool). After centrifugation, supernatants were collected and incubated with Protein A/G PLUSAgarose (Santa Cruz Biotechnology Inc., sc-2003) and $5 \mu$ g of the corresponding antibodies (TRAF3 antibody, Santa Cruz Biotechnology Inc., sc-6933; mouse IgG, Santa Cruz Biotechnology Inc., sc-2025) for 6 hours at $4^{\circ} \mathrm{C}$, followed by washing 5 times with IP buffer. Immunoprecipitated components were eluted by boiling in $1 \%$ (wt/vol) SDS buffer (60 mM Tris-HCl [pH 6.8], 1\% [wt/vol] SDS, 5\% [vol/vol] glycerol, 0.005\% [wt/vol] bromophenol blue, and 1\% [vol/ vol] 2-mercaptoethanol) for 10 minutes. For immunoblot analysis, immunoprecipitates and input lysates were separated by SDS-PAGE, followed by transferring onto PVDF membranes, and detected by specific antibodies.

Ubiquitination analysis. For polyubiquitination analysis of TRAF3 in HEK293T cells, HEK293T cells were transfected with plasmids expressing WT HECTD3 or mutant HECTD3 (C823A) and HAubiquitin (WT), HA-ubiquitin (K48), HA-ubiquitin (K63), HA-ubiquitin mutant (K63R), HA-ubiquitin (K6), HA-ubiquitin (K11), HA-ubiquitin (K27), HA-ubiquitin (K29), HA-ubiquitin (K33), HA-ubiquitin (K0, all lysine residues were mutated) or HA-ubiquitin (K48) with MG132, and Flag-TRAF3 (WT and mutants). Whole cell extracts were immunoprecipitated with the FLAG-specific antibody and analyzed by immunoblot with anti-HA and specific antibodies.

Lentivirus production and infection. HECTD3, WT TRAF3, and TRAF3 mutants were cloned into the lentiviral expression vector pCDH-CMV-MCS-EF1-Puro. The viral particles were prepared by transfecting HEK293T cells with HECTD3- and TRAF3-expressing or control plasmids in combination with packaging vectors. Twelve hours later, medium was replaced with fresh complete DMEM. Viral supernatant was harvested and passed through a $0.45 \mu \mathrm{m}$ syringe filter at 48 and 72 hours after transfection. To establish stably infected cells, WT, Hectd3 $3^{--}$, and TRAF3 $3^{-/-}$MEFs were infected with lentivirus as indicated in the presence of polybrene $(8 \mu \mathrm{g} / \mathrm{ml}) 3$ times. The infected cells were cultured in fresh media for at least 3 days prior to poly(I:C) stimulation and analysis.

ELISA. Cell culture supernatant and tissue samples or sera from uninfected and infected mice were analyzed for cytokine and chemokine release using ELISA MAX Standard (BioLegend) following the manufacturer's instructions.

Statistics. Data are shown as mean \pm SEM. Statistical analyses were performed using 1-way ANOVA with multiple comparisons, 2-tailed Student's $t$ test, and log-rank test. $P \leq 0.05$ was considered significant.

Study approval. All animal experiments were conducted in accordance with the guidelines of and were approved by the Kunming Institute of Zoology Animal Care and Use Committee.

Accession codes. All original microarray data were deposited in the NCBI's Gene Expression Omnibus database (GEO GSE104127).

\section{Author contributions}

XQ and CC designed the study. FL, YL, HL, TX, YK, MH, JX, X Chen, HX, YW, ZZ, XG, CH, CY, X Cheng, CC, and XQperformed experiments and analyzed the data. $\mathrm{XQ}$ and $\mathrm{CC}$ wrote the manuscript.

\section{Acknowledgments}

We thank C. Gao (Shandong University School of Basic Medical Sciences, Jinan, China), H.B. Shu (Wuhan University, Wuhan, China), and H. Xiao (Institut Pasteur of Shanghai, Chinese Academy of Sciences, Shanghai, China) for TBK1, STING, and TRIF plasmids; Y. Qian (Institute of Health Sciences, Shanghai Institutes for Biological Sciences, Shanghai, China) for Traf3 $3^{-/-}$MEFs; 
X. Zhang (Institut Pasteur of Shanghai) for GFP-expressing $M$. bovis BCG and regular BCG strains; J. Zhou (Kunming Institute of Zoology) for HSV-1; and S.Z. Duan (Institute for Nutritional Sciences, Shanghai Institutes for Biological Sciences) for L929 cells. This work was supported by grants from National Key Research and Development Program of China (2017YFD0500300), the Strategic Priority Research Program of the Chinese Academy of Sciences (XDA12010303), the National Natural Science Foundation of China (81325016,
U1602221, 31771516, 81701578, 31701134, 81402206, 81773149, and 81672639), and the Chinese Academy of Sciences (CXJJ17-M141, Y4ZK111B01, and Y602381081).

Address correspondence to: Ceshi Chen or Xiaopeng Qi, Kunming Institute of Zoology, Chinese Academy of Sciences, 32 East Jiaochang Road, Kunming, Yunnan 650223, China. Phone: 86.871.65181944; Email: chenc@mail.kiz.ac.cn (C. Chen). Phone: 86.871.65110289; Email: qixiaopeng@mail.kiz.ac.cn (X. Qi).
1. Tanwar J, Das S, Fatima Z, Hameed S. Multidrug resistance: an emerging crisis. Interdiscip Perspect Infect Dis. 2014;2014:541340.

2. Nikaido $H$. Multidrug resistance in bacteria. Annu Rev Biochem. 2009;78:119-146.

3. Boxx GM, Cheng G. The roles of type I interferon in bacterial infection. Cell Host Microbe. 2016;19(6):760-769.

4. Ivashkiv LB, Donlin LT. Regulation of type I interferon responses. Nat Rev Immunol. 2014;14(1):36-49.

5. Takeuchi O, Akira S. Pattern recognition receptors and inflammation. Cell. 2010;140(6):805-820.

6. Uematsu S, Akira S. Toll-like receptors and type I interferons. J Biol Chem. 2007;282(21):15319-15323.

7. Seth RB, Sun L, Ea CK, Chen ZJ. Identification and characterization of MAVS, a mitochondrial antiviral signaling protein that activates NF- $\kappa \mathrm{B}$ and IRF 3. Cell. 2005;122(5):669-682.

8. Wu J, Chen ZJ. Innate immune sensing and signaling of cytosolic nucleic acids. Annu Rev Immunol. 2014;32:461-488.

9. Sun L, Wu J, Du F, Chen X, Chen ZJ. Cyclic GMP-AMP synthase is a cytosolic DNA sensor that activates the type I interferon pathway. Science. 2013;339(6121):786-791.

10. Li S, Wang L, Berman M, Kong YY, Dorf ME. Mapping a dynamic innate immunity protein interaction network regulating type I interferon production. Immunity. 2011;35(3):426-440.

11. Metzger MB, Pruneda JN, Klevit RE, Weissman AM. RING-type E3 ligases: master manipulators of E2 ubiquitin-conjugating enzymes and ubiquitination. Biochim Biophys Acta. 2014;1843(1):47-60.

12. Bernassola F, Karin M, Ciechanover A, Melino G. The HECT family of E3 ubiquitin ligases: multiple players in cancer development. Cancer Cell. 2008;14(1):10-21.

13. Scheffner M, Kumar S. Mammalian HECT ubiquitin-protein ligases: biological and pathophysiological aspects. Biochim Biophys Acta. 2014;1843(1):61-74.

14. Rotin D, Kumar S. Physiological functions of the HECT family of ubiquitin ligases. Nat Rev Mol Cell Biol. 2009;10(6):398-409.

15. Tao J, Zhou X, Jiang Z. cGAS-cGAMP-STING: The three musketeers of cytosolic DNA sensing and signaling. IUBMB Life. 2016;68(11):858-870.

16. Heaton SM, Borg NA, Dixit VM. Ubiquitin in the activation and attenuation of innate antiviral immunity. J Exp Med. 2016;213(1):1-13.

17. Maelfait J, Beyaert R. Emerging role of ubiquitination in antiviral RIG-I signaling. Microbiol Mol
Biol Rev. 2012;76(1):33-45.

18. Song G, et al. E3 ubiquitin ligase RNF128 promotes innate antiviral immunity through K63linked ubiquitination of TBK1. Nat Immunol. 2016;17(12):1342-1351.

19. Wang C, et al. The E3 ubiquitin ligase Nrdp1 'preferentially' promotes TLR-mediated production of type I interferon. Nat Immunol. 2009;10(7):744-752.

20. Liu B, et al. The ubiquitin E3 ligase TRIM31 promotes aggregation and activation of the signaling adaptor MAVS through Lys63linked polyubiquitination. Nat Immunol. 2017;18(2):214-224.

21. Gack MU, et al. TRIM25 RING-finger E3 ubiquitin ligase is essential for RIG-I-mediated antiviral activity. Nature. 2007;446(7138):916-920.

22. Kuniyoshi K, et al. Pivotal role of RNA-binding E3 ubiquitin ligase MEX3C in RIG-I-mediated antiviral innate immunity. Proc Natl Acad Sci US A. 2014;111(15):5646-5651.

23. Lang X, Tang T, Jin T, Ding C, Zhou R, Jiang W. TRIM65-catalized ubiquitination is essential for MDA5-mediated antiviral innate immunity. J Exp Med. 2017;214(2):459-473.

24. Tsuchida T, et al. The ubiquitin ligase TRIM56 regulates innate immune responses to intracellular double-stranded DNA. Immunity. 2010;33(5):765-776.

25. Zhang J, Hu MM, Wang YY, Shu HB. TRIM32 protein modulates type I interferon induction and cellular antiviral response by targeting MITA/ STING protein for K63-linked ubiquitination. J Biol Chem. 2012;287(34):28646-28655.

26. Wang $Q$, et al. The E3 ubiquitin ligase AMFR and INSIG1 bridge the activation of TBK1 kinase by modifying the adaptor STING. Immunity. 2014;41(6):919-933.

27. Qin Y, et al. RNF26 temporally regulates virus-triggered type I interferon induction by two distinct mechanisms. PLoS Pathog. 2014;10(9):e1004358.

28. Arimoto K, Takahashi H, Hishiki T, Konishi $\mathrm{H}$, Fujita T, Shimotohno K. Negative regulation of the RIG-I signaling by the ubiquitin ligase RNF125. Proc Natl Acad Sci U S A. 2007;104(18):7500-7505.

29. Zhong B, et al. The ubiquitin ligase RNF5 regulates antiviral responses by mediating degradation of the adaptor protein MITA. Immunity. 2009;30(3):397-407.

30. Wang Y, et al. TRIM30 $\alpha$ is a negative-feedback regulator of the intracellular DNA and DNA virus-triggered response by targeting STING. PLoS Pathog. 2015;11(6):e1005012.
31. Cui J, et al. NLRP4 negatively regulates type I interferon signaling by targeting the kinase TBK1 for degradation via the ubiquitin ligase DTX4. Nat Immunol. 2012;13(4):387-395.

32. Zhang M, et al. TRAF-interacting protein (TRIP) negatively regulates IFN- $\beta$ production and antiviral response by promoting proteasomal degradation of TANK-binding kinase 1. JExp Med. 2012;209(10):1703-1711.

33. Wang $\mathrm{P}$, Zhao W, Zhao K, Zhang L, Gao C. TRIM26 negatively regulates interferon- $\beta$ production and antiviral response through polyubiquitination and degradation of nuclear IRF3. PLoS Pathog. 2015;11(3):e1004726.

34. Li Y, et al. The HECTD3 E3 ubiquitin ligase suppresses cisplatin-induced apoptosis via stabilizing MALT1. Neoplasia. 2013;15(1):39-48.

35. Li Y, et al. The HECTD3 E3 ubiquitin ligase facilitates cancer cell survival by promoting K63-linked polyubiquitination of caspase-8. Cell Death Dis. 2013;4:e935.

36. Silva MT, Correia-Neves M. Neutrophils and macrophages: the main partners of phagocyte cell systems. Front Immunol. 2012;3:174.

37. Hall JD, et al. Infected-host-cell repertoire and cellular response in the lung following inhalation of Francisella tularensis Schu S4, LVS, or U112. Infect Immun. 2008;76(12):5843-5852.

38. Meunier E, et al. Guanylate-binding proteins promote AIM2 inflammasome activation during Francisella novicida infection by inducing cytosolic bacteriolysis and DNA release. Nat Immunol. 2015;16(5):476-484.

39. Man SM, et al. The transcription factor IRF1 and guanylate-binding proteins target activation of the AIM2 inflammasome by Francisella infection. Nat Immunol. 2015;16(5):467-475.

40. Qi X, et al. Cathepsin B modulates lysosomal biogenesis and host defense against Francisella novicida infection. JExp Med. 2016;213(10):2081-2097.

41. Storek KM, Gertsvolf NA, Ohlson MB, Monack DM. cGAS and Ifi204 cooperate to produce type I IFNs in response to Francisella infection. J Immunol. 2015;194(7):3236-3245.

42. Oganesyan G, et al. Critical role of TRAF3 in the Toll-like receptor-dependent and -independent antiviral response. Nature. 2006;439(7073):208-211.

43. Abe T, Barber GN. Cytosolic-DNA-mediated, STING-dependent proinflammatory gene induction necessitates canonical NF- $\kappa \mathrm{B}$ activation through TBK1. J Virol. 2014;88(10):5328-5341.

44. Wang G, et al. K63-linked ubiquitination in kinase activation and cancer. Front Oncol. 2012;2:5. 
45. Tseng PH, Matsuzawa A, Zhang W, Mino T, Vignali DA, Karin M. Different modes of ubiquitination of the adaptor TRAF3 selectively activate the expression of type I interferons and proinflammatory cytokines. Nat Immunol. 2010;11(1):70-75.

46. Miao Y, Wu J, Abraham SN. Ubiquitination of innate immune regulator TRAF3 orchestrates expulsion of intracellular bacteria by exocyst complex. Immunity. 2016;45(1):94-105.

47. Henry T, et al. Type I IFN signaling constrains IL-17A/F secretion by $\gamma \delta \mathrm{T}$ cells during bacterial infections. J Immunol. 2010;184(7):3755-3767.

48. Lin WW, Hostager BS, Bishop GA. TRAF3, ubiq- uitination, and B-lymphocyte regulation. Immunol Rev. 2015;266(1):46-55.

49. Zinngrebe J, Montinaro A, Peltzer N, Walczak H. Ubiquitin in the immune system. EMBO Rep. 2014;15(1):28-45.

50. Liu S, et al. MAVS recruits multiple ubiquitin E3 ligases to activate antiviral signaling cascades. Elife. 2013;2:e00785.

51. Fang DF, et al. NEDD4 ubiquitinates TRAF3 to promote CD40-mediated AKT activation. Nat Commun. 2014;5:4513.

52. Huai W, et al. Mint3 potentiates TLR3/4- and RIG-I-induced IFN- $\beta$ expression and antiviral immune responses. Proc Natl Acad Sci US A.
2016;113(42):11925-11930.

53. Chen HW, Yang YK, Xu H, Yang WW, Zhai ZH, Chen DY. Ring finger protein 166 potentiates RNA virus-induced interferon- $\beta$ production via enhancing the ubiquitination of TRAF3 and TRAF6. Sci Rep. 2015;5:14770.

54. Qi X, et al. Antagonistic regulation by the transcription factors C/EBP $\alpha$ and MITF specifies basophil and mast cell fates. Immunity. 2013;39(1):97-110.

55. Qi X, et al. Critical role of caspase-8-mediated IL-1 signaling in promoting Th2 responses during asthma pathogenesis. Mucosal Immunol. 2017;10(1):128-138. 